\title{
One-dimensional cell motility patterns
}

\author{
Jonathan E. Ron, ${ }^{1}$ Pascale Monzo $\odot,{ }^{2}$ Nils C. Gauthier, ${ }^{2}$ Raphael Voituriez, ${ }^{3}$ and Nir S. Gov ${ }^{1}$ \\ ${ }^{1}$ Department of Chemical and Biological Physics, Weizmann Institute of Science, 7610001, Israel \\ ${ }^{2}$ IFOM, FIRC Institute of Molecular Oncology, Milan, 20139, Italy \\ ${ }^{3}$ Laboratoire Jean Perrin and Laboratoire de Physique Thorique de la Matire Condense, Sorbonne Universit, \\ Tour 13-12, 5eme etage, 4 place Jussieu, 75252 Paris Cedex 05, France
}

(Received 6 March 2020; accepted 23 July 2020; published 11 August 2020)

\begin{abstract}
During migration, cells exhibit a rich variety of seemingly random migration patterns, which makes unraveling the underlying mechanisms that control cell migration a difficult challenge. For efficient migration, cells require a mechanism for polarization, so that traction forces are produced in the direction of motion, while adhesion is released to allow forward migration. To simplify the study of this process, cells have been studied when placed along one-dimensional tracks, where single cells exhibit both smooth and stick-slip migration modes. The stick-slip motility mode is characterized by protrusive motion at the cell front, coupled with a slow elongation of the cell, which is followed by a rapid retraction of the cell rear. In this study, we explore a minimal physical model that couples the force applied on the adhesion bonds to the length variations of the cell and to the traction forces applied by the polarized actin retrograde flow. We show that the rich spectrum of cell migration patterns emerges from this model as different deterministic dynamical phases. This result suggests a source for the large cell-tocell variability $(\mathrm{CCV})$ in cell migration patterns observed in single cells over time and within cell populations: fluctuations in the cellular components, such as adhesion strength or polymerization activity, can shift the cells from one migration mode to another, due to crossing the dynamical phase transition lines. Temporal noise is shown to drive random changes in the cellular polarization direction, which is enhanced during the stick-slip migration mode. The model contains an emergent critical length for cell polarization, whereby cells that retract below this length loose polarity, and are prone to making direction changes in migration. These results offer a new framework to explain experimental observations of migrating cells, resulting from noisy switching between underlying deterministic migration modes.
\end{abstract}

DOI: 10.1103/PhysRevResearch.2.033237

\section{INTRODUCTION}

Eukaryote cell migration, whereby cells crawl actively over an external substrate, is a subject of great interest for biological processes such as development and cancer progression. Adhesion-based cell motility involves the orchestration of a large number of cytoskeletal proteins: Typically, the cell needs to break its symmetry (polarize), and produce traction forces in the direction of polarization (balanced by drag or friction forces). The traction forces are mediated by adhesion between the cell and the external substrate, but these adhesions have to detach at the trailing end of the cell in order to allow the cell to migrate forward. When observing freely migrating cells (i.e., not guided by an external gradient of any kind), one is often struck by the large variation in their migration patterns [1-5], both for a single cell over time, and for a (seemingly identical) cell population. The origin of the large cell-to-cell variability $(\mathrm{CCV})$, or phenotypic, population heterogeneity,

Published by the American Physical Society under the terms of the Creative Commons Attribution 4.0 International license. Further distribution of this work must maintain attribution to the author(s) and the published article's title, journal citation, and DOI. observed during cell migration is not understood [6], and is usually ascribed to the inherent noise of cellular systems [7,8].

To simplify the study of the complex process of cell motility, cells can be confined to move along one-dimensional tracks, either on flat adhesive stripes $[9,10]$, linear grooves [11] and channels [12,13], or thin fiber [14,15]. In addition to being a simple geometry for the study and analysis of the cell motion in general, such confined motion appears also in-vivo [16], for example when cells move along axonal fibers $[17,18]$, or cancer invades in confined spaces between tissues [19].

The experiments listed above have shown that isolated cells on one-dimensional tracks exhibit the following stereotypical behaviors [9,10]: (i) nonmigrating and unpolarized, by remaining quiescent or elongating symmetrically, (ii) undergoing spontaneous symmetry breaking, polarization and migrating smoothly, and (iii) as in (ii) but exhibiting stick-slip migration. The stick-slip motility mode is characterized by protrusive motion at the cell front, coupled with an overall elongation of the cell and followed by rapid retraction of the cell back. In both (ii) and (iii), the cell motility can be highly persistent, or undergo sporadic direction changes. The appearance of this variety of migration behaviors within a uniform cell population, as well as the switching of cells between these modes, remains an open puzzle and is the aim 
of this work. Clearly the diversity of the migration patterns is beyond modeling the cell motility as a simple random walk process $[4,20-22]$.

We present a theoretical model that describes the cell motility in a highly simplified, and coarse-grained manner. By coarse-grained we mean that we do not describe the cellular dynamics at the molecular or actin-filament scale. Nevertheless, the model contains two key, and strongly coupled, components: a slip-bond adhesion module at the cell back and a cellular polarization module. We find that these components are sufficient to drive the entire spectrum of observed motility patterns, and explain the transitions between them. Our work therefore demonstrates how a minimal model gives rise to a rich variety of deterministic migration patterns, as opposed to complex motion that is purely driven by different levels of cellular noise. In experiments, these deterministic patterns may underlie the migration of cells, but further confounded by noise.

The stick-slip motion is shown here to have an underlying deterministic oscillatory behavior, separated from smooth migration by a bifurcation line. Previous treatments of stick-slip dynamics of cells focused on the protrusion-retraction cycles and the adhesion dynamics at the cell edge [23-25] but did not include a mechanism for the polarization of the cytoskeleton activity between the front and the back of the motile cell. Here, we couple the stick-slip adhesion at the cell back to the overall cell polarization, through the dependence of the polarization on the cell length. This dependence arises in our model due to the dependence of the polarized actin flow on the gradient of an advected polarity cue. Below a critical length, the polarity cue can not sustain a sufficient gradient to maintain the polarized actin flow that advects it. This is an inherent property of the UCSP model [26] that was not previously explored. Deterministic oscillations in the speed of migrating dendritic cells, for example, were related to competition for finite resources that directly affected the actomyosin polarization mechanism, but did not involve length oscillations [27]. Furthermore, the dendritic-cell oscillations depend on a specific macropinocytosis process, while here we obtain deterministic oscillatory (stick-slip) migration patterns that are driven by adhesion dynamics that are much more general across cell types. We compare the results of the model to new experiments carried out on glioma cells, which are both highly motile and are naturally migrating along onedimensional-like substrates in vivo [28,29].

\section{MODEL}

The model is introduced by three parts, with increasing levels of complexity and realism. In this manner, we expose the motility patterns and the key components that drive them.

The first part describes a cell that is constantly polarized, with a constant protrusive activity at the leading edge, and slip bond adhesions at the rear [24]. This part allows us to expose the oscillatory stick slip behavior through the dynamics of the cell length and adhesion concentration at the rear.

The second part adds a self-polarization model to the polarized cell [26], i.e., having a single leading edge. This part couples the dynamics in cell length to the protrusive activity, and introduced a critical polarization length scale.

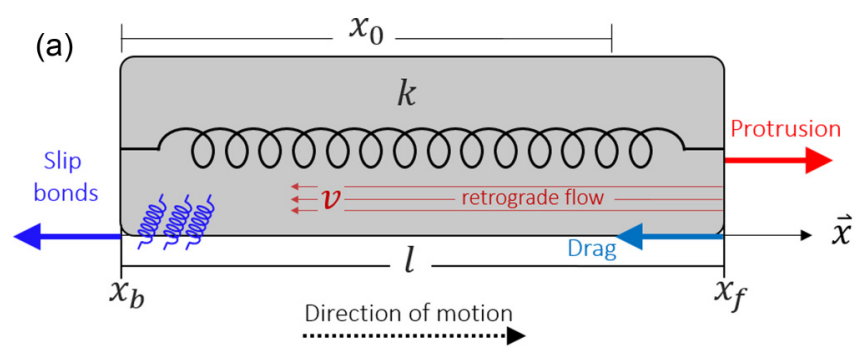

(c)

(b)
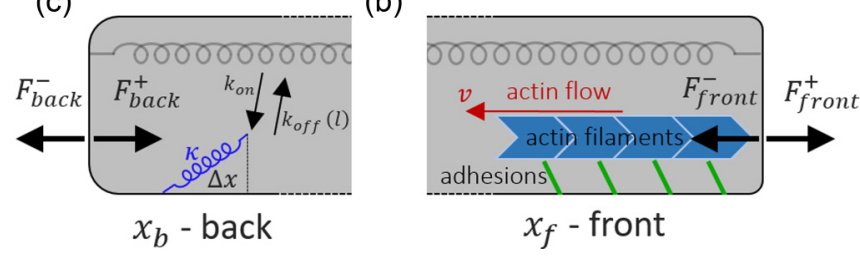

FIG. 1. The simplified model. (a) Illustration of the physical model of a cell migrating along a linear track. $x_{b}$ and $x_{f}$ represent the back and front part of the cell of the cell which are connected by an effective spring with a stiffness $k . x_{0}$ is the rest length of the spring (cell) and $l$ is the length of the cell. $v$ is the velocity of the actin retrograde flow which is assumed to be constant. At the front acts a protrusion force (red arrow) and a drag force (teal arrow). At the back acts a friction force due to the slip bonds (blue arrow). (b) The physical model of the stick slip adhesion at the back $x_{b}$. Stochastic linkers with stiffness $\kappa$ attach with an average rate of $k_{\text {on }}$ and detach with a length dependent rate of $k_{\text {off }}(l)$ at the back [Eq. (8)]. The linkers stretch on average with a displacement of $\Delta x$ [Eq. (10)]. $F_{\text {back }}^{ \pm}$ [Eqs. (5) and (6)] are the forces that act on the trailing edge of the cell. (c) The physical model of the protrusion force acting at the front $x_{f} . F_{\text {front }}^{ \pm}$[Eqs. (1) and (2)] are the forces that act on/from the sliding actin filaments, which are balanced by catch adhesions (green short lines).

In the third part, the model is extended to be symmetric, such that the protrusion and adhesion dynamics acts on both edges of the cell. This part outlines the conditions for symmetry breaking and the role of noise in choosing a migration direction.

\section{A. Polarized cell with a constant protrusion}

\section{Model description}

Consider a cell of length $l$ that migrates along a linear track. The two ends of the cell, the front and back, denoted by $x_{f}$ and $x_{b}$, are connected by a spring [Fig. 1(a)]. The stiffness of the spring $k$, represents the effective elasticity of the cell cytoplasm and membrane. Such a mechanical coupling between the front and rear was recently demonstrated experimentally [30].

In this part of the model, the motion of the cell is considered to be already polarized, such that the actin treadmilling from the front to the back occurs at a constant velocity $v$, and produces a constant protrusive force at the front

$$
F_{\text {front }}^{+}=\alpha v,
$$

where $\alpha$ describes the strength of the coupling of the actin retrograde flow and the effective friction force generated by adhesions which grip the sliding filaments. This is a simplified representation of the "clutch" mechanism [23], that converts 
the sliding of the actin to a protrusive force that pushes on the membrane. This coupling is dependent on the adhesion strength, and we therefore expect a term of the form: $r /(r+$ $r_{0}$ ) to multiply the right-hand side (r.h.s.) of Eq. (1), where $r$ is the ratio between the binding/unbinding rates of the cellsubstrate adhesion molecules $r=k_{\text {on }} / k_{\text {off }}^{0}$, and $r_{0}$ quantifies the cell-substrate adhesion saturation. Such a term accounts for the loss of traction force when the adhesion diminishes $r \rightarrow 0$. We do not explicitly describe here the catch-bond property of these adhesions, as we are not interested in the stick-slip dynamics of the leading edge, but rather wish to focus on the stick-slip events on the whole cell scale. For the rest of this paper we effectively work in the limit of $r_{0} \ll r$ (the effects of $r_{0}$ are shown in Fig. 23).

The pushing force is balanced by a drag force which is proportional to the speed of the moving front, and a restoring force due to the global cell elasticity

$$
F_{\text {front }}^{-}=\gamma \dot{x}_{f}+k\left(x_{f}-x_{b}-x_{0}\right),
$$

where $\gamma$ represents the effective resistance to the motion of the cell front due to the friction generated by the contact of adhesion molecules with the substrate. As in Eq. (1), we expect a term of the form $r /\left(r+r_{0}\right)$ to multiply the first term on the r.h.s. of Eq. (2), but we neglect it by choosing to work in the limit of $r_{0} \ll r$. The parameter $k$ represents the stiffness of the spring, which describes the effective elasticity of the cell, and the parameter $x_{0}$ represents the rest length of the cell.

Equating (1) and (2) due to the force balance at $x_{f}$ yields

$$
\alpha v=\gamma \dot{x}_{f}+k\left(x_{f}-x_{b}-x_{0}\right),
$$

which allow to obtain the equation of motion for the moving cell front

$$
\dot{x}_{f}=\frac{1}{\gamma}\left(\alpha v-k\left(x_{f}-x_{b}-x_{0}\right)\right) .
$$

At the back, $x_{b}$, the pulling force due to the cell elasticity

$$
F_{\text {back }}^{+}=k\left(x_{f}-x_{b}-x_{0}\right)
$$

is balanced by a friction force which results from the stretching of bound linkers, which model slip bond adhesions

$$
F_{\text {back }}^{-}=n \kappa\langle\Delta x\rangle,
$$

where $n$ is the mean number of bound linkers, $\kappa$ is the spring constant of the linkers, and $\langle\Delta x\rangle$ is the average displacement of the linkers [Fig. 1(c)]. The number of bound linkers evolves dynamically and obeys the following kinetics:

$$
\dot{n}=k_{\text {on }}(N-n)-k_{\text {off }} n,
$$

where $N$ is the total number of linkers, $k_{\text {on }}$ is the basal attachment rate, and $k_{\text {off }}$ is a detachment rate which depends exponentially on the force applied on each bound linker $[23,24]$

$$
k_{\text {off }}=k_{\text {off }}^{0} \exp \left(\frac{f_{l}}{f_{s}}\right),
$$

where $k_{\text {off }}^{0}$ is the basal detachment rate and $f_{s}$ represents the susceptibility of the linkers to the applied force [24]. The stretching force per bound linker is

$$
f_{l}=\frac{k\left(x_{f}-x_{b}-x_{0}\right)}{n} .
$$

The average stretch of each linker $\langle\Delta x\rangle$ depends on the dissociation rate of the linkers [(8) and (7)] and can be written as

$$
\langle\Delta x\rangle=\frac{\dot{x}_{b}}{k_{\text {off }}} .
$$

By combining (8)-(10) into the force balance between (5) and (6), we obtain

$$
n \kappa \frac{\dot{x}_{b}}{k_{\text {off }}^{0} \exp \left(\frac{k\left(x_{f}-x_{b}-x_{0}\right)}{n f_{s}}\right)}=k\left(x_{f}-x_{b}-x_{0}\right),
$$

where $\kappa$ is the effective spring constant of the linkers, and we substituted for the average stretching of each linker: $\langle\Delta x\rangle=\dot{x}_{b} / k_{\text {off }}$. By reorganizing (11), we obtain the equation of motion of the moving back

$$
\dot{x}_{b}=\frac{k\left(x_{f}-x_{b}-x_{0}\right)}{n \kappa} k_{\text {off }}^{0} \exp \left(\frac{k\left(x_{f}-x_{b}-x_{0}\right)}{n f_{s}}\right) .
$$

Note that we treat the adhesions at the front and back of the cell differently. The motivation for this difference arises from the way that the forces are applied to these adhesion sites [31]; at the front, the adhesion molecules have actin flow over them and they undergo a nucleation-maturation process, while at the back they undergo detachment due to mechanical pulling (and possibly other biochemical degradation processes). Adhesions at advancing edges of the cell have been found to behave differently from those at the trailing edges [31].

Combining (4) and (12) and changing coordinates to $l=$ $x_{f}-x_{b}$ yields the following dynamical system:

$$
\begin{gathered}
i=\frac{\alpha}{\gamma} v-k\left(l-x_{0}\right)\left[\frac{1}{\gamma}-\frac{k_{\text {off }}^{0}}{n \kappa} \exp \left(\frac{k\left(l-x_{0}\right)}{n f_{s}}\right)\right], \\
\dot{n}=k_{\text {on }}(N-n)-k_{\text {off }} n .
\end{gathered}
$$

Next, the system [(13) and (14)] is rescaled by the time and length scales of $1 / k_{\text {off }}^{0}, x_{0}$, and by the total number of adhesion sites $N$, as well as rescaled by the parameters $\frac{v}{x_{0} k_{\text {off }}^{0}} \rightarrow$

$$
\begin{gathered}
\tilde{v}, \frac{k}{k_{\mathrm{off}}^{0}} \rightarrow \tilde{k}, \frac{f_{s} N}{x_{0} k_{\mathrm{off}}^{0}} \rightarrow \tilde{f}_{s}, \frac{N \kappa}{k_{\mathrm{off}}^{0}} \rightarrow \tilde{\kappa}, \text { and } \frac{k_{\mathrm{on}}^{0}}{k_{\mathrm{off}}^{0}} \rightarrow r \text { to obtain } \\
i=\frac{\alpha}{\gamma} \tilde{v}-\tilde{k}(l-1)\left[\frac{1}{\gamma}-\frac{1}{\tilde{\kappa} n} \exp \left(\frac{\tilde{k}(l-1)}{\tilde{f}_{s} n}\right)\right] \\
\dot{n}=r(1-n)-n \exp \left(\frac{\tilde{k}(l-1)}{\tilde{f}_{s} n}\right)
\end{gathered}
$$

Finally, we normalize the force scale and simplify the analysis by setting $\alpha=1$ and $\gamma=1$, and remove the tilde signs in Eqs. (15) and (16), to obtain

$$
\begin{gathered}
\dot{l}=v-k(l-1)\left(1-\frac{\exp \left(\frac{k(l-1)}{f_{s} n}\right)}{\kappa n}\right), \\
\dot{n}=r(1-n)-n \exp \left(\frac{k(l-1)}{f_{s} n}\right) .
\end{gathered}
$$

Note that the dynamics is now captured by two ODEs, where the spatial component appears only as the total cell length variable. These reduced equations have four structural parameters $\left(r, k, \kappa, f_{s}\right)$ and the parameter describing the strength of the actin treadmilling flow $(v)$. For a detailed discussion of the choice of model parameters used in the paper, we refer the reader to Appendix A. 

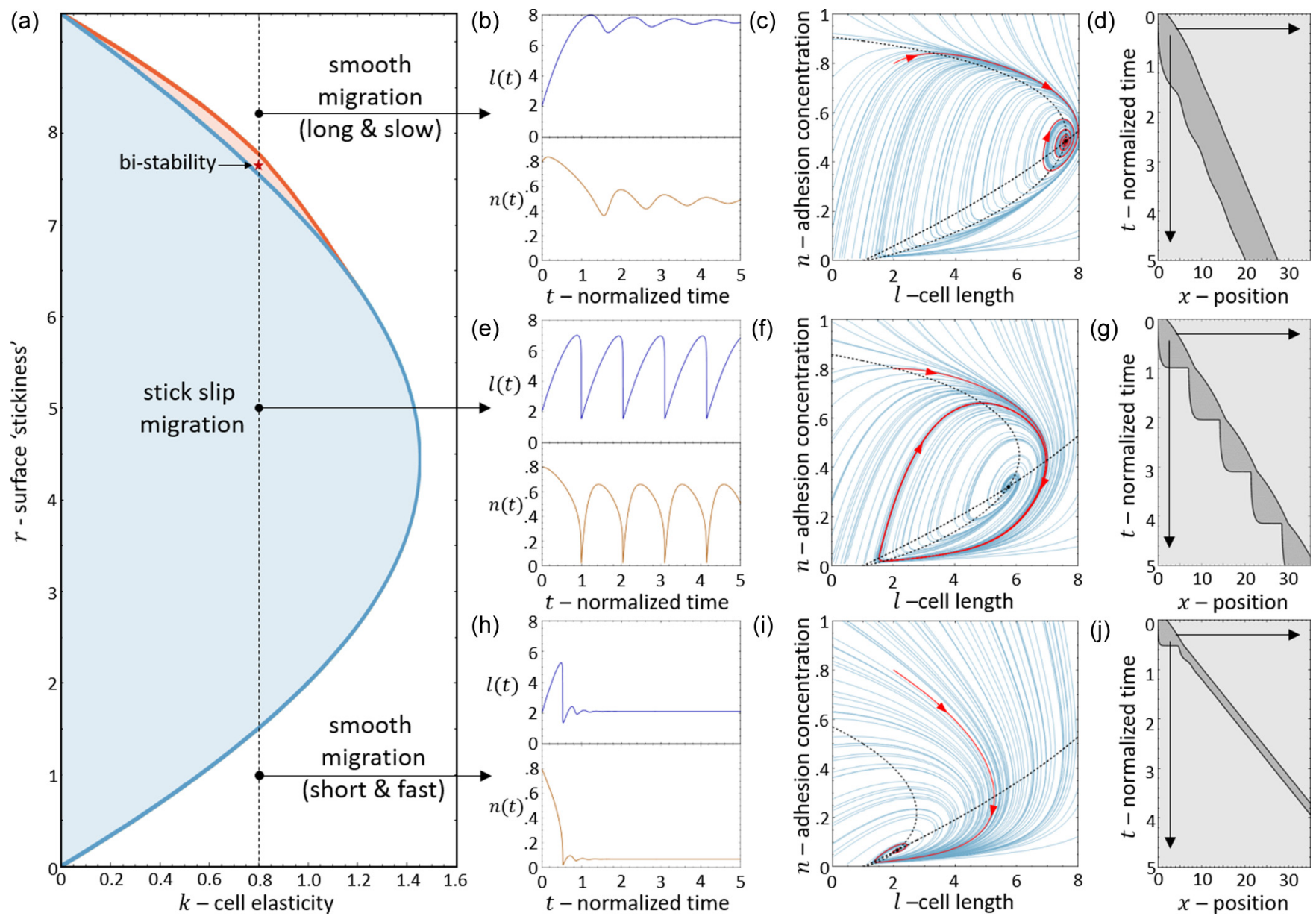

FIG. 2. (a) $k-r$ phase diagram. Blue region correspond to the polarized stick-slip cells. White region corresponds to polarized smooth migrating cells with a constant length. Red region corresponds to bistability between polarized cells with a constant length and polarized stick slip migrating cells. Blue/Red curves are the Hopf/saddle node bifurcation transition lines. Black dashed line is the $r$ cross section for $k=0.8$. Black dots correspond to the points $(k, r)=0.8,1 ;(k, r)=0.8,5$; and $(k, r)=0.8,8.4$. Red star correspond to the point $(k, r)=(0.8,7.7)$. [(b)-(d)] The dynamics at $(k, r)=0.8,1$. [(b)-(d)] The dynamics at $(k, r)=0.8,5$. [(h)-(i)] The dynamics at $(k, r)=0.8,1$. Blue/orange curves in (b), (e), and (h)correspond to the time series of the cell length and adhesion concentration at the rear. Red curve in (c), (f), and (i) represent the trajectory in $l, n$ phase space. Black curves in (d), (g), and (i) are the kymographs where the black curves are cell edges $x_{b}$ and $x_{f}$. Parameters: $f_{s}=5, \kappa=20$, and $v=10$.

\section{Results}

It is convenient to plot the resulting dynamics on the $k-r$ phase diagram shown in Fig. 2(a). We find that above a critical value of the cell stiffness $k$ (to the right of the blue solid line), the spring is too stiff to allow for large length changes that enables the spring to store and release large forces. In this regime, we get a single, stable fixed point that corresponds to smooth cell motion, and no stick-slip behavior (for the phase diagram as function of the adhesion saturation parameter $r_{0}$ see Appendix B).

Below the critical stiffness, we find that for small values of $r$, the system corresponds to a smooth motion (stable fixed point) of a short and fast-moving cell [Figs. 2(h)-2(j)].

Above a transition line [solid blue line in Fig. 2(a)], the fixed point undergoes a Hopf bifurcation, which marks the transition from smooth motion to stick-slip motion (limitcycle dynamics) [Figs. 2(e)-2(g)]. In this regime, there are large length oscillations, as well as large oscillations in the number of bound adhesion linkers at the cell back [Figs. 3(a) and 3(b)]. These oscillations in $n$ occur due to the large values of the force per linker that is reached [Fig. 3(c)], which leads to catastrophic, avalanche-like detachment events at the back. Within the stick-slip regime, we find that the duration of the limit-cycle increases with increasing $r$, i.e., the dynamics slow down with increasing substrate adhesiveness [Fig. 3(d)].

As $r$ increases there is a second Hopf bifurcation, whereby the fixed point is stable again. For large values of $r$ the adhesion at the back is stable, as it can sustain the pulling force exerted by the traction forces at the front. The cell is therefore stretched, and the back slides smoothly, exerting a large friction that slows down the migration. In addition, there is a narrow region of bistability, where the phase space is separated by a separatrix [solid black line in Fig. 3(b), such that the stable fixed point coexists with the limit cycle. Within this regime, noise can induce transitions from stick-slip (limit 

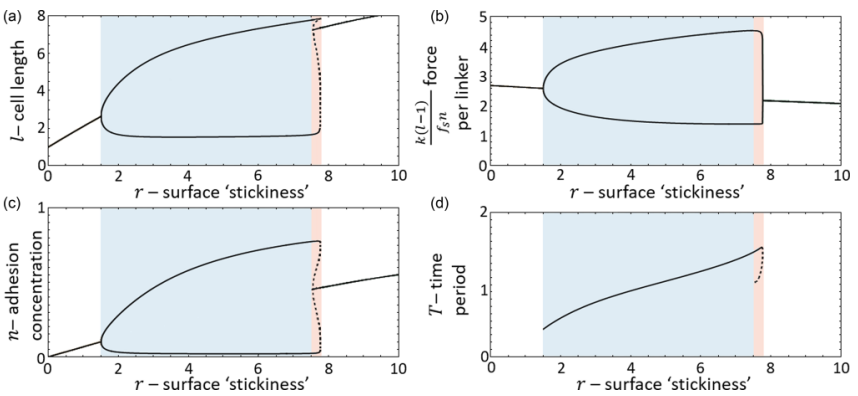

FIG. 3. Stability analysis along the line $k=0.8$ [vertical black dashed line in Fig. 2(a)]. (a) The maximal and minimal length as a function of $r$. (b) The maximal and minimal force applied on a linker at the back part of the cell as a function of $r$. (c) The maximal and minimal adhesion concentration at the rear as a function of $r$. (d) The time period of the limit cycle as a function of $r$. Solid black curves indicate the stable limit cycle. Dashed black curves indicate the unstable limit cycle. Parameters: $v=10, \kappa=20$, and $f_{s}=5$.

cycle) to smooth motion (stable fixed point), as demonstrated in Fig. 4.

For increasing $r$, the separatrix grows until it meets the limit cycle, which marks the transition to flows that all lead to the single stable fixed point. The dynamics in this regime correspond to a smooth motion of a slow moving and elongated cell [Figs. 2(b)-2(d) and 3].

\section{Comparison to experiments}

We now compare in detail the model's stick-slip limit-cycle dynamics [Figs. 2(e)-2(g)] to experiments (see Appendix C for experimental methods). In Fig. 5, we analyze the stick-slip migration mode of a C6 glioma cell (seeded on laminin-coated lines of $5 \mu \mathrm{m}$ width), by following the cell length and the intensity of membrane-bound vinculin, using total internal reflection fluorescence (TIRF) microscopy. As a component of the adhesion complex, we denote the total intensity of vinculin in the rear of the nucleus as a measure of the adhesion strength at the cell back in the model $(n)$. We find that the experimentally observed dynamics during a stick-slip cycle give rise to a limit cycle in the $n, l$-phase space that has the same qualitative features as the model predicts [Fig. 2(f)].

The robust features of the migration modes predicted by the model can be observed in experiments (Fig. 6). In Fig. 6(a), we show a kymograph of a motile cell moving along a one-dimensional stripe, exhibiting stick-slip behavior. At a certain point along the trajectory, the cell increased both its rate of stick-slip events, and its overall migration velocity. In addition, the amplitude of the stick-slip events decreased in size. All of these features are captured by the model, if the average adhesion strength between the cell and the substrate $(r)$ decreases abruptly along the trajectory, and the cell moves from high to low $r$ within the stick-slip regime [Fig. 2(a)].

In Fig. 6(b), we show a cell migrating smoothly, and then abruptly decreasing in length and increasing in speed. This behavior is captured by the model assuming that the adhesion strength $r$ decreases such that the cell jumps from high to low $r$ as shown in Figs. 2(a), 2(d) and 2(j).

Note that we can not exclude that the abrupt change in behavior observed for the cells in Fig. 6 originates from a
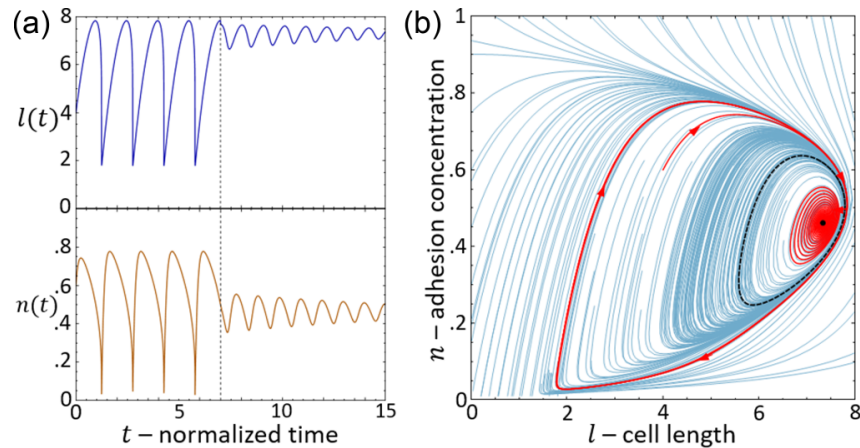

(c)

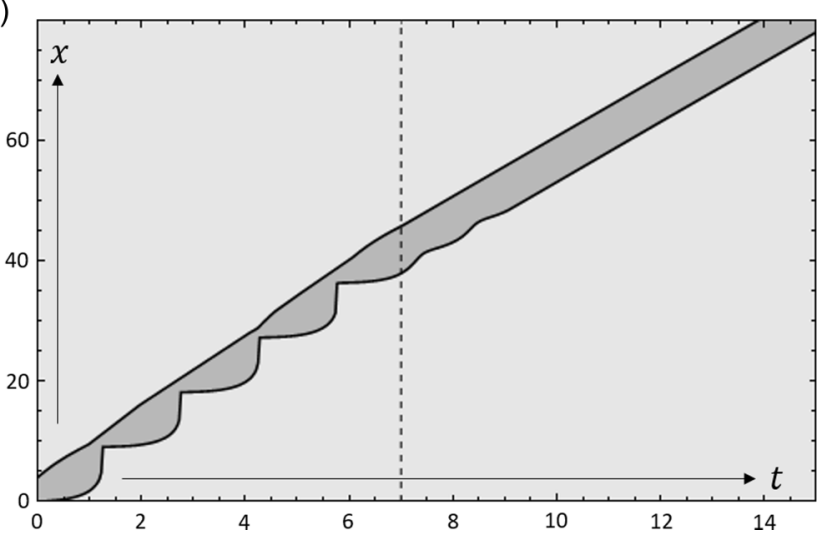

FIG. 4. [(a)-(c)] Dynamics in the bistability regime [red star in Fig. 2(a). (a) Blue and orange curves are the time series of the cell length and adhesion concentration at the rear. Black dashed line indicates the time when noise was injected into the solution of the differential equation [Eq. (17)]. (b) $n-l$ phase diagram. Red curve is the trajectory and black curve is the separatrix between the two modes of motion. (c) The corresponding kymograph: black curves represent the edges of the cell $x_{b}$ and $x_{f}$. Dashed black line indicates the time when noise was injected. Parameters: $v=10, k=0.8, \kappa=$ 20 , and $f_{s}=5$.

change in some other internal parameter of the cell. Nevertheless, recent experiments [32] indicate that these glioma cells are highly sensitive to the adhesion strength, which strongly affects their migration behavior, as our model suggests. A previous work [15] showed that dynamic changes in the cell-substrate adhesion, as exposed by vinculin fluorescence levels, can transform a cell from being stalled (i.e., extending in both directions but remaining unpolarized) to motile. This demonstrates that cells are poised close enough to the transition between these different modes, and naturally occurring fluctuations in the adhesion strength are enough to move them across the transition line.

Cell migration has been shown to be dramatically affected by changes in substrate composition [9]. In Fig. 24 (Appendix D), we provide a direct measure of the heterogeneities in the surface coverage by laminin along the onedimensional stripes, which occur during the preparation of these stripes. In particular, there are high concentration puncta deposited along the track, and moving across them could initiate abrupt changes in the cell-substrate adhesion strength.

Note that the backwards growth of the lamellipodia at the cell back [Fig. 6(a)], during each stick-slip cycle, are described by the more elaborate model below. In addition, 
(a)
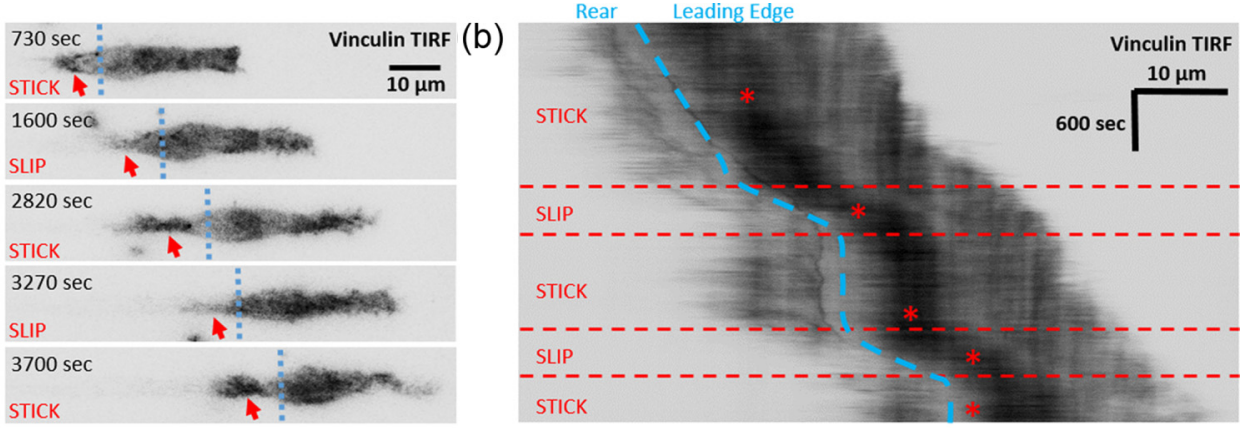

(c)

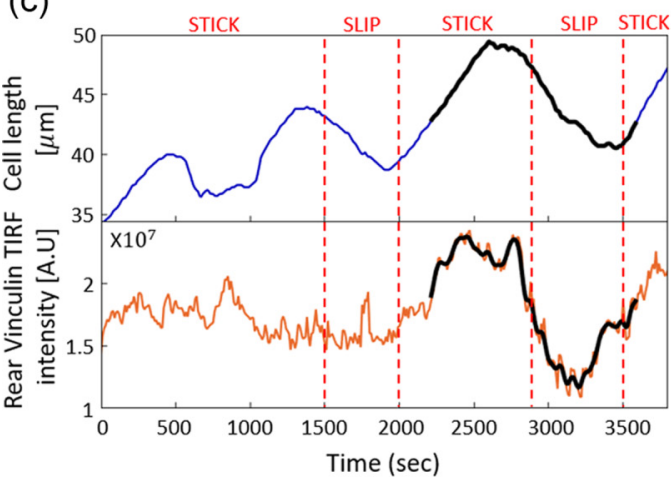

(d)

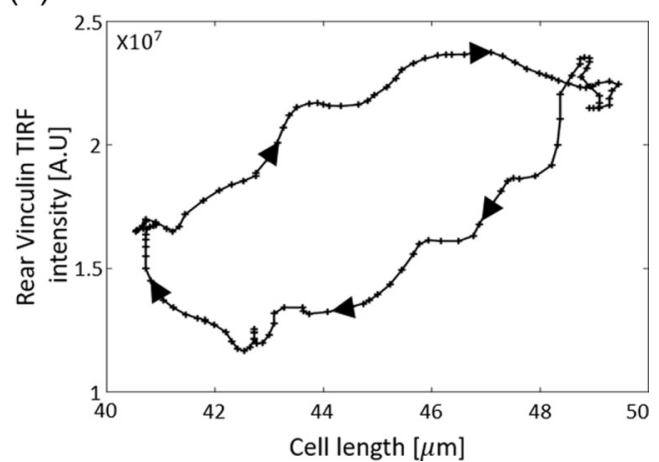

FIG. 5. Comparison of the basic stick-slip model [Eqs. (17)and (18)] to the stick-slip dynamics observed in experiments (patient-derived Human glioma propagating cell NNI21 transfected with fluorescently tagged vinculin and seeded on laminin-coated lines of $5 \mu \mathrm{m}$ width and imaged every $110 \mathrm{sec}$ ). (a) Movie snap shots, where the cells have fluorescently labeled vinculin (represents the extent of adhesion complexes). The region that defines the cell back is to the left of the vertical dashed line, i.e., behind the nucleus. (b) A kymograph of the cell migration, with the stick slip events marked. (c) The dynamics of the cell length and the total amount of vinculin signal at the cell back region, as function of time. The black line on both graphs indicates the stick-slip cycle used to plot the phase-space limit cycle shown in (d).

we observe highly dynamic stick-slip behavior at the leading edge of the cell, which are also associated with propagating actin waves [15], and which we do not describe by our current model $[23,33]$.

\section{B. Polarized cell with a dynamic protrusion}

\section{Model description}

We now complement the length-adhesion model described above, by incorporating a model for the spontaneous self- polarization of the cell. We use a modified version of the scheme developed in Ref. [26] [Fig. 7(a)], whereby in addition to the advection of a "polarity-cue" protein that enhances the actin treadmilling, such as myosin-II, we also consider a polarity-cue that acts as an inhibitor of actin polymerization. The inhibitor protein is free to diffuse in the cytoplasm, and is also advected by the actin treadmilling flow. An example for an inhibitor of local actin polymerization, that is advected by the actin flow, is Arpin [26,34]. We furthermore assume that the timescale of the redistribution of the polarity-cue protein (a)

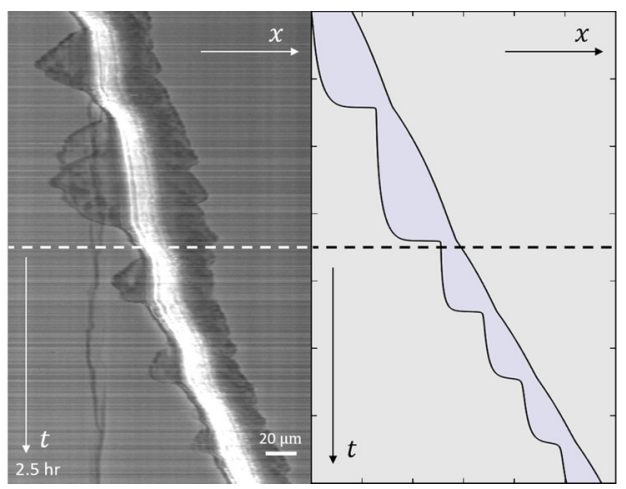

(b)

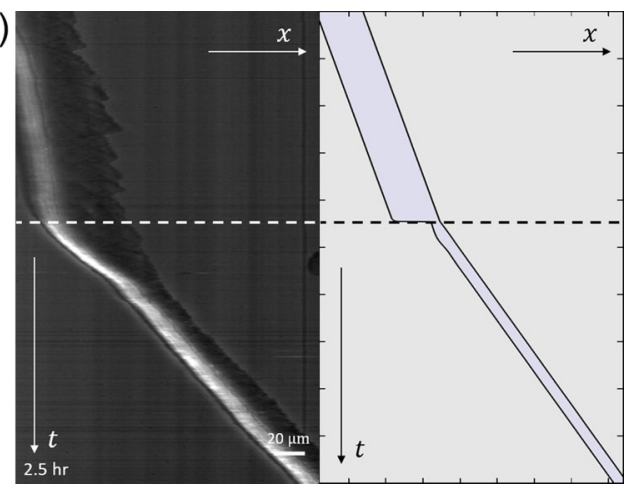

FIG. 6. Comparison of the basic stick-slip model [Eqs. (17) and (18)] to experiments on C6 glioma cells seeded on laminin-coated lines of $5 \mu \mathrm{m}$ width (imaged every $30 \mathrm{sec}$ ). Kymographs correspond to total time 2.5 hours. (a) Comparison to model results with $r=7$, then abruptly changed to $r=3$ (indicated by the horizontal dashed line). (b) As in (a), with $r=8.5$ then changing abruptly to $r=1$. Model parameters: $v=10, k=0.8, \kappa=20$, and $f_{s}=5$. 


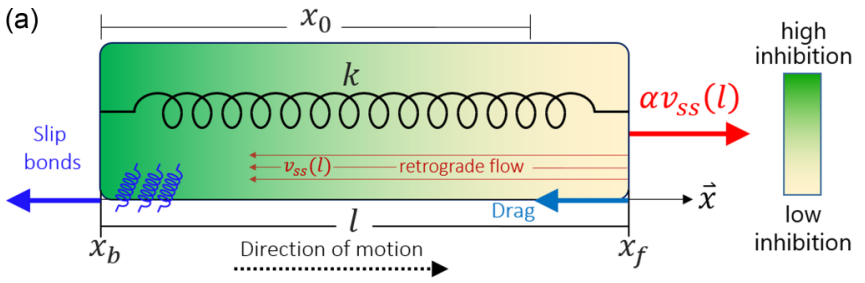

(b)

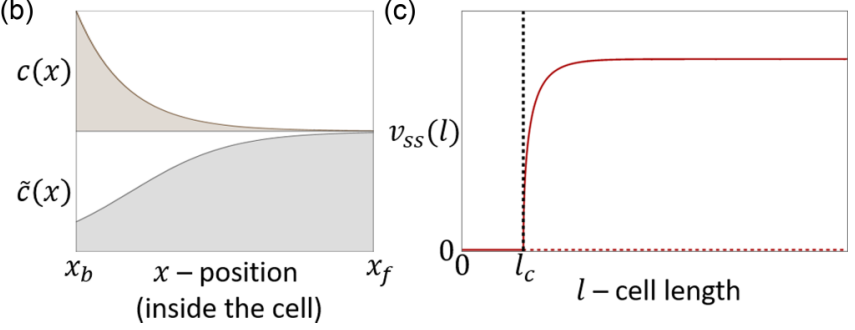

FIG. 7. The stick-slip UCSP model. (a) Illustration of a the physical model. Unlike the simple model of Fig. 1, the treadmilling velocity is now dependent on the cell length $v(l)$, due to the advection of an inhibitory cue. The concentration of the inhibitory cue is represented by the colorbar on the right. The steady-state concentration of the inhibitory cue, $c(x)$, is shown in [(b), top], which is an exponential function due to a balance between diffusion and advection [Eq. (19)]. The effect of $c(x)$ on the local actin polymerization rate is given by a Hill function [Eq. (20)] [(b), bottom]: where the inhibitory cue concentration is low the local actin polymerization rate is large. (c) The steady-state retrograde flow speed as a function of cell length [solution of Eq. (21)]. Red solid line represent the stable solution. Red dashed line represent the unstable solution. Black dashed line notes the critical length $l_{c}$ of polarization.

(inhibitor) across the cell is much shorter than the timescale of changes in the actin treadmilling speed. This assumption, of separation of timescales, was also made in Ref. [26], and is corroborated by recent measurements of myosin-II redistribution time when advected by actin $(\sim 10 \mathrm{sec})$ [30], while the stick-slip cycles of the cell are over timescales of tens of minutes [9].

Another key assumption which we make is of quasistatic boundaries when calculating the profile of the polarization cue across the length of the cell, $c(x)$ [in Eq. (19) below and derived in Appendix E]. Here, we work under the assumption that the motion of the cell boundaries (i.e., its length changes) are slow compared to the redistribution of $c(x)$ along the cell. Specifically, this amounts to the regime where the motion of the cell boundaries is slow compared to the actin flow, such that the rate of cell-length change obeys $i \ll v$ [see Eqs. (E6) and (E7)]. Within this regime, we can solve the steady-state $c(x)$ considering the boundaries to be static and solving at each time according to the instantaneous length and the treadmilling actin flow $v$. The timescale of the stick-slip cycle is of tens of minutes, and this is longer than the timescale over which we expect the polarity cue to redistribute within the cell. For example, a polarity cue that enhances local actin treadmilling, myosin-II, was recently observed to redistribute on a much shorter timescale of tens of seconds [30], and therefore supports the validity of our approximation. However, during a slip event, the cell does change its length rapidly, on the order of tens of seconds. During these very short periods, our approximation of quasistatic boundary when calculating the polarity cue distribution is not valid. Nevertheless, these periods extend over a very small fraction of the cell migration, and we do not expect them to be significantly affect the overall validity of our results.

We can therefore use the steady-state distribution of the concentration of this inhibitor $c(x)$, which is given by an exponential function [Fig. 7(b)]

$$
c(x)=\frac{c_{\mathrm{tot}} v}{D}\left(\frac{e^{-\frac{v x}{D}}}{e^{-\frac{v x_{b}}{D}}-e^{-\frac{v x_{f}}{D}}}\right)
$$

where $v$ is the effective instantaneous actin treadmilling velocity, $D$ the effective diffusion coefficient of the inhibitor, and $c_{\text {tot }}$ is the total amount of inhibitor molecules in the cytoplasm (see Appendix E).

To complete the model [26], we need to relate the treadmilling flow of actin across the cell to the polarity cue gradient. In Refs. [26,27], the treadmilling flow was assumed to be dominated by the imbalance in the contractile forces of myosin-II across the cell length, and myosin-II was treated as the polarity cue. Here we rather focus on the actin flow due to polymerization activity at the two opposing ends of the cell. We therefore treat $c(x)$ as an inhibitor of actin polymerization, but similarly one could augment this by including an additional polarity cue that affects the treadmilling flow due to myosin-II contractility. We focus in this work only on the contribution of the polymerization activity. The net treadmilling flow is therefore given by the difference between the actin polymerization flows created at the two ends of the cell, which are inhibited by the local concentration of the inhibitor at the front and rear

$$
v\left(x_{f}, x_{b}\right)=\beta\left(\tilde{c}\left(x_{f}\right)-\tilde{c}\left(x_{b}\right)\right),
$$

where $\beta$ gives the scale of the actin flow in the cell, and the polymerization activity at each end is diminished by the inhibitor concentration at each end, given by a Hill function [26,27]: $\tilde{c}\left(x_{b, f}\right)=c_{s} /\left(c_{s}+c\left(x_{b, f}\right)\right)$ [Fig. 7(b)], where $c_{s}$ is the concentration at which the effect of the inhibitor saturates.

After rescaling (see Appendix F), using Eqs. (19) and (20), we obtain the following implicit equation for the net actin treadmilling flow:

$$
v(l)=\beta\left(\frac{1}{1+c \frac{v}{D}\left(\frac{1}{e^{\frac{v l}{D}}-1}\right)}-\frac{1}{1+c \frac{v}{D}\left(\frac{1}{1-e^{-\frac{v l}{D}}}\right)}\right) .
$$

In the persistent regime (sufficiently large $c_{s}$ ), we find that as $l$ increases above a critical value Eq. (21) undergoes a pitchfork bifurcation, where spontaneous actin treadmilling appears, corresponding to a polarized and motile cell [Fig. 7(c)]. The critical length is given by

$$
l_{c}=\frac{c}{\sqrt{\frac{c \beta}{D}-1}}
$$

which provides a lower bound for the coupling strength $\beta>$ $D / c$, below which there is no spontaneous motility. A lower critical length for polarization was also obtained in Ref. [35]. 


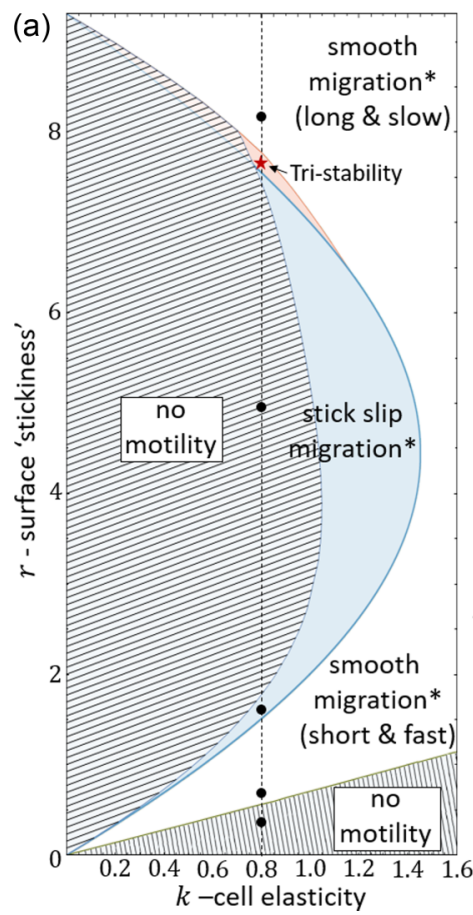

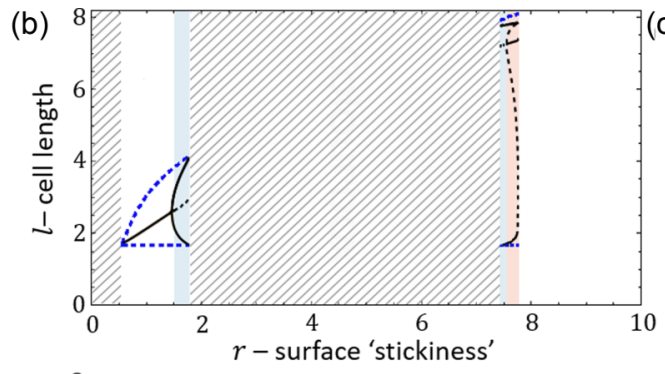

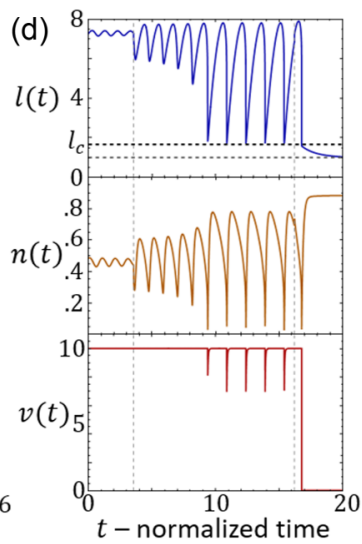

(e)
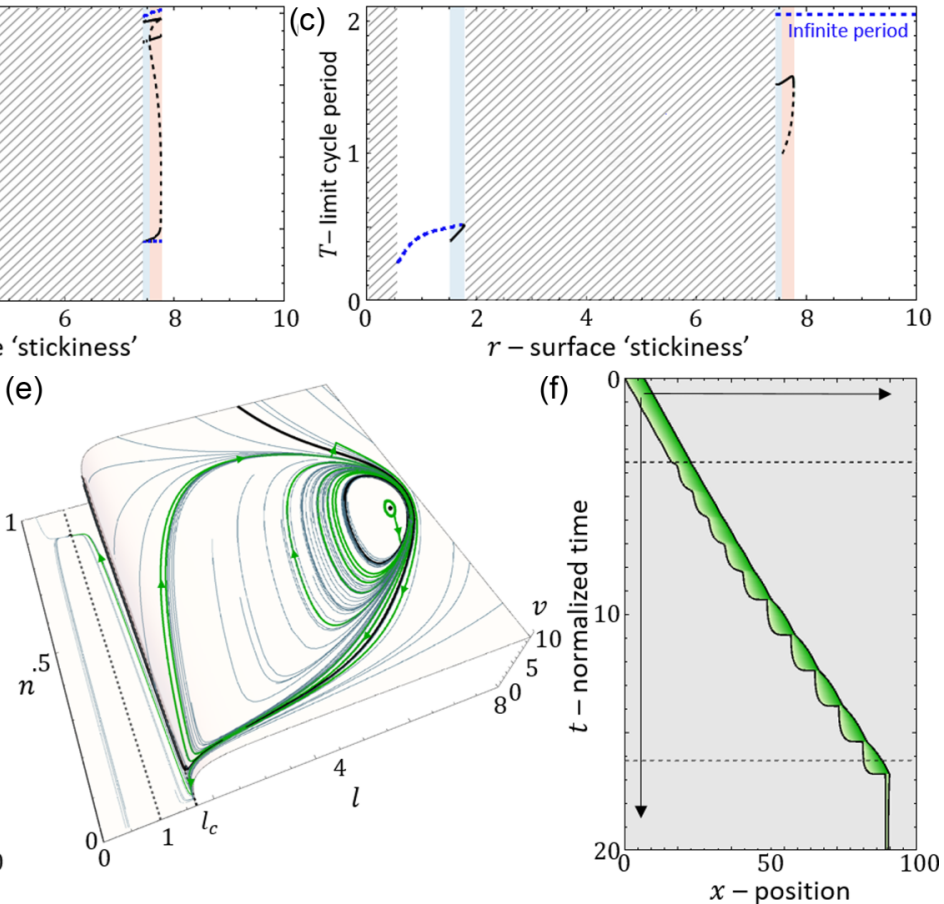

FIG. 8. (a) $k-r$ phase diagram. Striped texture regions correspond to regimes where there is no motility (stationary cell). Blue region corresponds to a bistable region of polarized stick-slip cells and stationary cells. White region corresponds to bistability between stick slip and smooth migration. Red region corresponds to tristability between stick slip, smooth migration, and stationary cells. Blue/red/brown curves are the Hopf/saddle node/Hopf bifurcation transition lines. Black dashed line is the $r$ cross section for $k=0.8$. Black dots correspond to the points $(k, r)=(0.8,0.4),(0.8,0.7),(0.8,1.65),(0.8,5),(0.8,8)$. Red star correspond to the point $(k, r)=(0.8,7.7)$. (b) Maximal and minimal amplitude of the $l$ state variable as a function of $r$ for the limit cycles in the vector field along the cross section of $k=0.8$. Dashed blue curve correspond to the unstable discontinuous limit cycle. Solid black curve corresponds to the stable limit cycle and the cell length. Black dashed curve is the unstable limit cycle. (c) The time period of the limit cycles. Blue dashed line indicate the discontinuous unstable limit cycle. Black solid line indicate the stable limit cycle (period of stick slip). Black dashed line indicates the unstable limit cycle in the tri-stable region. [(d)-(f)] The tristable region. Solution of Eqs. (23) and (24) with noise of amplitude $(\delta l, \delta v)=(0.5,0.11)$ injected at $t=3.55$ and $(\delta l, \delta n)=(0.5,0.05)$ injected at $t=16.2$, to demonstrate the transition between the phases (across the separatrix lines). Blue/orange/red curves in (d) correspond to the dynamics of length/adhesion concentration and actin retrograde flow. Gray dashed lines indicate the time point in which the noise was injected. Green curve in (e) is the trajectory in the $l-n-v$ phase space. Black solid lines are the separatrices. (f) displays the kymograph which corresponds to [(d) and (e)]. Parameters: $f_{s}=5, \kappa=20, \beta=11, c=3.85$, and $D=3.85$.

The equations of motion are now extended beyond the basic model [Eqs. (17) and (18)] to include the dynamics of the actin treadmilling flow

$$
\begin{gathered}
i=v-k(l-1)\left(1+\frac{\exp \left(\frac{k(l-1)}{n f_{s}}\right)}{n}\right), \\
\dot{n}=r(1-n)-n \exp \left(\frac{k(l-1)}{n f_{s}}\right), \\
\dot{v}=-\delta\left(v-v_{s s}(l)\right),
\end{gathered}
$$

where $v_{s s}(l)$ is the steady-state solution to Eq. (21), which is the length-dependent actin flow speed, and $\delta$ is the rate at which the actin flow adjusts to length changes, i.e., the rate at which $v$ approaches $v_{s s}(l)$.

For a cell that has a rest length that is longer than the critical length for polarization, $l_{c}<1$, the resting state of the cell is polarized. Since the velocity saturates to a constant value for $l>l_{c}$ [Fig. 7(c)], the results are qualitatively similar to those we obtained for a constant, length-independent, $v$ (Fig. 3).

We will therefore focus on the more interesting case where $l_{c}>1$, and the rest length of the cell is smaller than the critical length for polarization. In this case, we find another fixed point that corresponds to $v=0$

$$
\left(l^{*}, n^{*}\right)=\left(1, \frac{r}{1+r}\right)
$$

where the cell is at its rest length and stationary.

\section{Results}

In Fig. 8(a), we plot the $k-r$ phase diagram for the simpler case of $\delta \rightarrow \infty$, such that the actin flow speed is given directly by the solution $v_{s s}(l)$ [Eq. (20), Fig. 7(c)]. In comparison with the case of fixed actin flow [Fig. 2(a)], there are several new phases, despite the overall similar shape of the phase diagram.

For very low values of $r$, we find that there is a transition line below which the cell is nonmotile [brown region in Fig. 8(a)], which corresponds to all the flows in the $n-l$ phase space leading to the single fixed point at $v=0$ [Eq. (26)]. Above this transition line, with increasing $r$, there are phases of coexistence between smooth motion or stick slip, and nonmotile behavior. We plot in Fig. 9 the dynamics for different points of increasing value of $r$, for fixed $k=0.8$ [vertical 

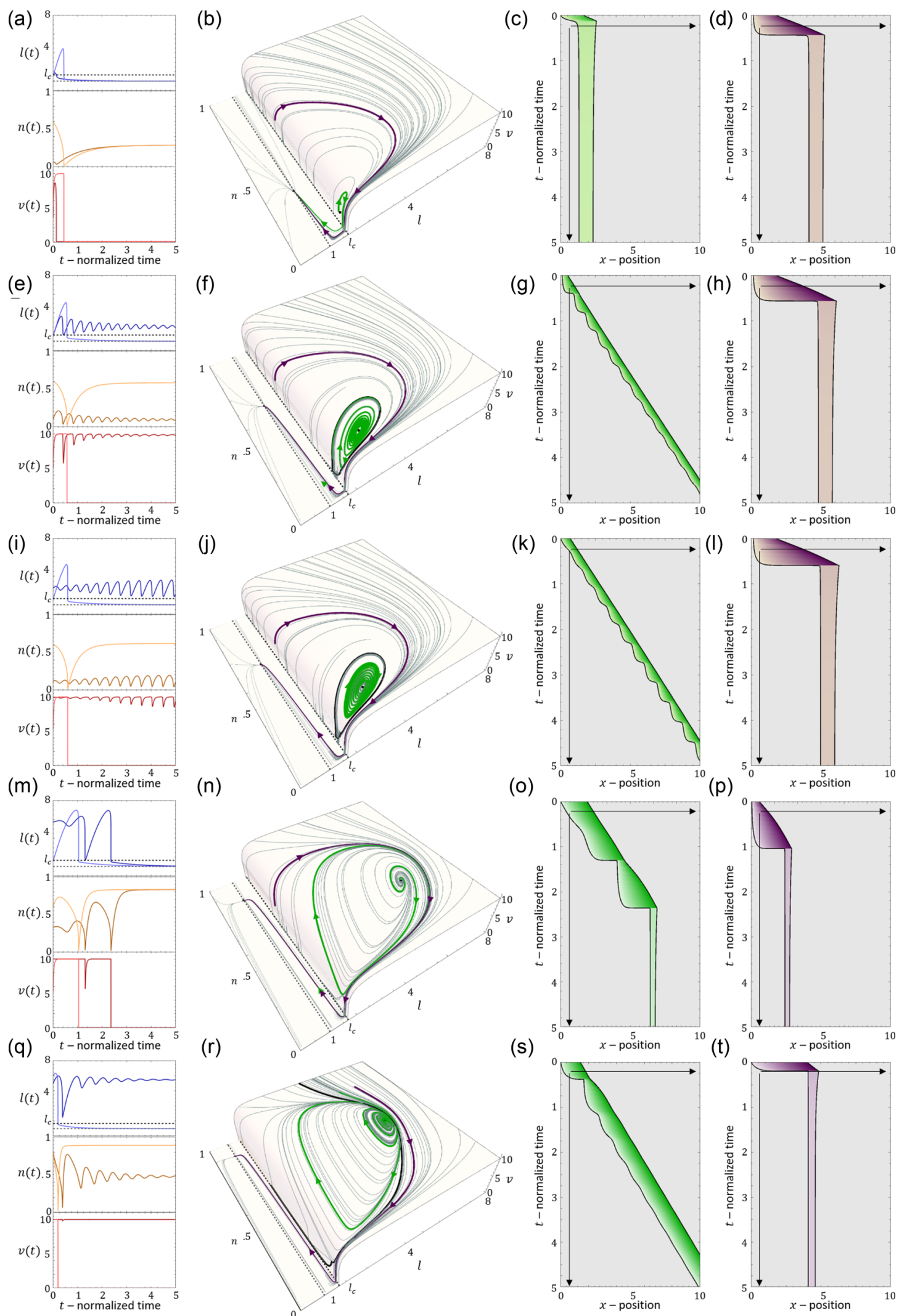

FIG. 9. The dynamics along the line of constant $k=0.8$ [vertical dashed line in Fig. 8(a)]. [(a)-(d)] $r=0.4$, [(e)-(h)] 0.7, [(i)-(l)] 1.65, $[(\mathrm{m})-(\mathrm{p})] 5$, and [(q)-(t)] $r=8$. Blue/orange/red curves in (a), (e), (i), (m), and (q) correspond to the time series of the cell length, adhesion concentration and actin retrograde flow respectively (bold/thin lines corresponds to green/purple trajectory). Green and purple lines (b), (f), $(\mathrm{j}),(\mathrm{n})$, and (r) demonstrate the trajectories in the bistable $l-n-v$ phase space regime. Black solid curves are the separatrices. (d), (g), and (i) display the corresponding kymographs. Parameters: $f_{s}=5, \kappa=20, \beta=11, c=3.85$, and $D=3.85$. 

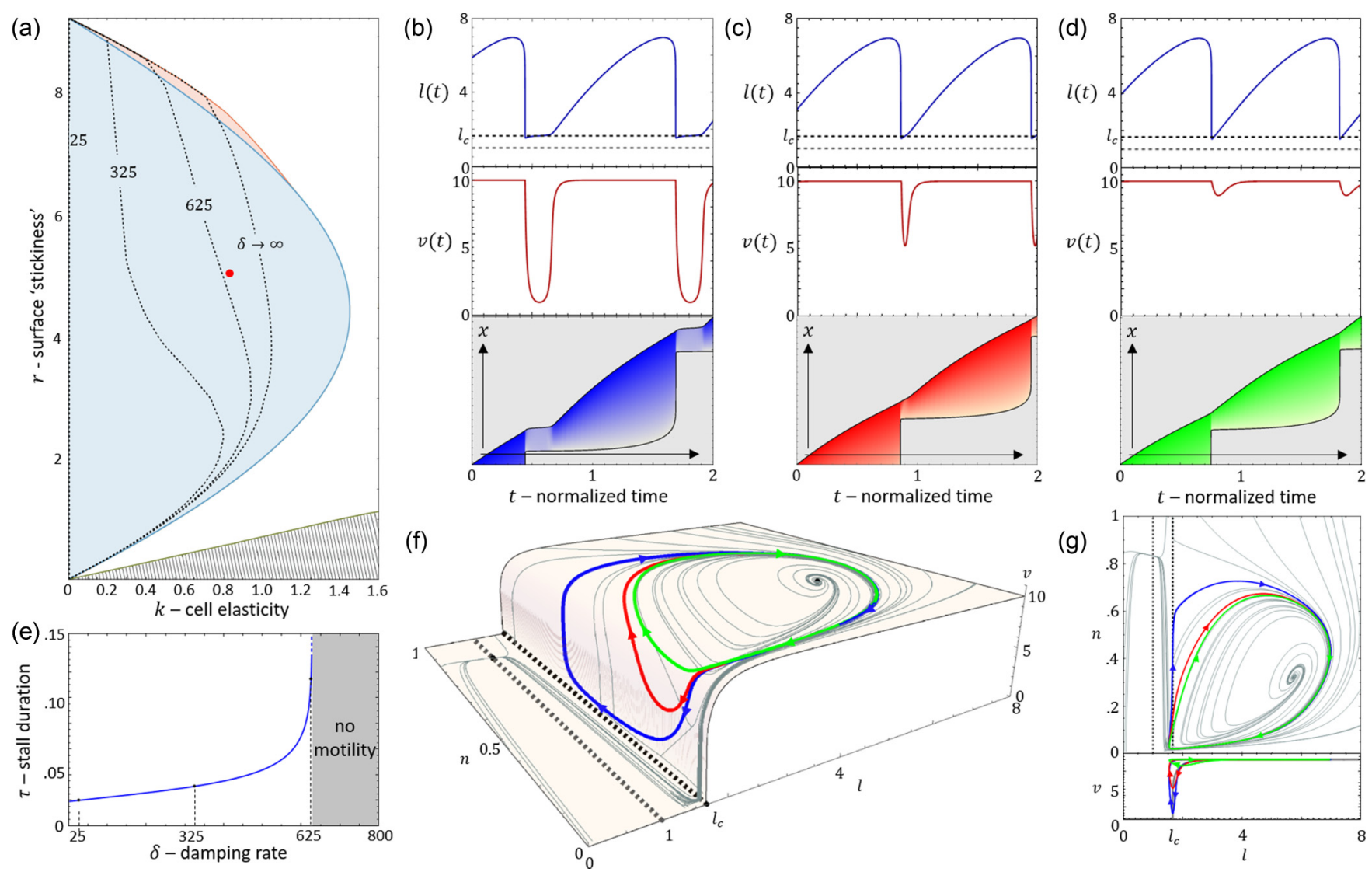

FIG. 10. The effect of a finite actin response rate $\delta$ on the dynamics [Eq. (25)]. (a) The $k$-r phase diagram for different values of $\delta$ [in Eq. (25)]. Dashed black lines indicate the coalescence of the discontinuous unstable and stable limit cycles, which produce the no-motility regime for different values of $\delta$. The phase transition line for $\delta \rightarrow \infty$ is the same as in Fig. 8(a). [(b)-(d)] Demonstration of the lagging time for values of $\delta$ at the point $(k, r)=(0.8,5)$ on the phase diagram (black dot). Blue and red curves are the time series of the cell length and actin retrograde flow speed, respectively. Black dashed line is the critical length of polarization and gray dashed line is the rest length of the cell. Blue, red, and green kymographs correspond to $\delta=625,325$, and 25 respectively. (e) The duration of the stall as a function of $\delta$, indicating the values of $\delta=625,325$, and 25 at the point $(k, r)=(0.8,5)$. Gray region indicates where there is no motility for every initial condition. (f) The stable limit cycles which correspond to (b)-(d) in the $l-n-v$ phase space. (g) Projections of the stable limit cycles which correspond to (b) $-(\mathrm{d})$ in the $l-n$ and $l-v$ phase spaces. Other parameters: $f_{s}=5, \kappa=20, \beta=11, c=3.85$, and $D=3.85$.

dashed line in Fig. 8(a)]. Two flows are demonstrated for each case (green and purple paths and corresponding kymographs in Fig. 9), exposing the coexistence of either smooth motion [(e)-(h) and (q)-(t)] or stick slip [(i)-(l)] with the nonmotile phase. The bifurcations of the stable and unstable solutions are denoted by their cell length (and the limit cycle amplitude), as well as the limit cycle periods, in Figs. 8(b) and 8(c) (along the same $k=0.8$ line).

There is even a thin region of tristability [Fig. 8(a)], which we demonstrate in Figs. 8(d)-8(f), by inducing a transition between the three phases by adding noise at specific times. For a complete exploration of the $k-r$ phase diagram see Appendix G.

Next, we explore the effect of a finite value of $\delta$, where the treadmilling velocity of the actin does not instantaneously adjust to its length-dependent value $v_{s s}(l)$ [Eq. (25)].

In Fig. 10(a), we plot the shift in the stick-slip transition line to lower values of $k$, for decreasing values of $\delta$. The region of no-motility is now pushed to lower values of $k$. The reason for this is shown in Figs. 10(b)-10(d): when the length drops below $l_{c}$ after the slip event, the velocity of the actin does not drop to zero instantaneously (as in the case when $\delta \rightarrow \infty$ ). There is therefore a region of the phase diagram where the length recovers and increases above $l_{c}$, allowing the stick-slip limit cycle to survive. The "stall duration," during which $l<$ $l_{c}$ and the cell is almost stalled, increases with increasing value of $\delta$ [Fig. 10(e)].

\section{Self-polarized symmetric cell with a dynamic protrusion}

\section{Model description}

In the model, we developed so far, we did not allow for the actin polymerization to produce local traction and protrusive forces at both ends, but rather assumed that the competition between the two ends gives rise to a single leading edge (or to an unpolarized cell) [Fig. 7(a)].

We now extend the model to describe the local protrusive forces that are produced by actin polymerization at the two ends of the cell [Fig. 11(a)].

The dynamics of the moving front and back depend on their respective direction of motion: when they protrude there is a drag force that is given by a constant drag coefficient, while 

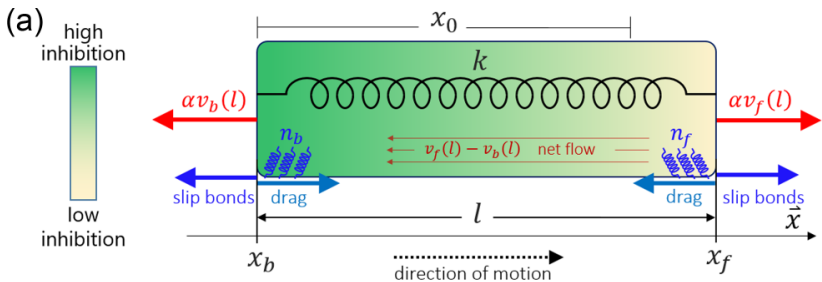

(b)

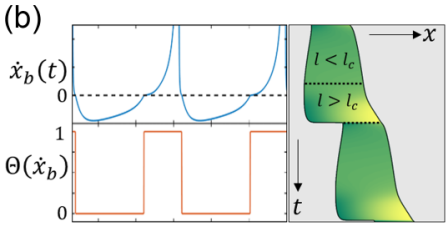

$t$ - normalized time

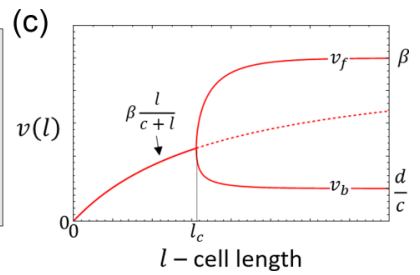

FIG. 11. The symmetric model. (a) Illustration of the physical model. $x_{b}$ and $x_{f}$ are the back and front part of the cell which are connected by a spring with a stiffness $k . x_{0}$ is the rest length of the spring and $l$ is the length of the cell. $v_{f}$ and $v_{b}$ are the steady-state velocities of actin retrograde flow from both ends of the cell, which are coupled to the gradient of an inhibitory cue. The concentration of the inhibitory cue is represented by the colorbar on the left. At both ends of the cell, three forces act: a protrusion force (red arrow), which is proportional to the retrograde flow speed (dark red arrow), a drag force (teal arrow) when the edge is moving forward, and a friction force due to the slip bonds (blue arrow) when the edge is moving backwards. (b) Demonstration of the friction model in Eq. (28): upper panel displays in blue the derivative of the moving rear $\dot{x}_{b}$. Below in orange is the Heaviside theta function of the time series of $\dot{x}_{b}$. Right panel displays the corresponding kymograph. (c) The steady-state retrograde flow speed as a function of cell length [Eq. (31)]. Red solid line represents the stable solution. Red dashed line represents the unstable solution. Black dashed line denotes the critical length of polarization $l_{c}$. Parameters: $\beta=19, c=9$, and $D=40$.

when they retract the friction depend on the slip-bond activity of the adhesion [Fig. 11(b)]. We therefore have the following equations of motion for the two ends of the cell:

$$
\dot{x}_{f / b}=\frac{1}{\Gamma_{f / b}}\left( \pm v_{f / b} \mp k\left(x_{f}-x_{b}-1\right)\right),
$$

where the friction coefficients are given by

$$
\begin{aligned}
\Gamma_{f / b}= & 1-\Theta\left(\mp \dot{x}_{f / b}\right) \\
& +\Theta\left(\mp \dot{x}_{f / b}\right) n_{f / b} \kappa \exp \left(\frac{v_{f / b}-k\left(x_{f}-x_{b}-1\right)}{f_{s} n_{f / b}}\right),
\end{aligned}
$$

where $\Theta$ is the theta Heaviside function. This way the friction coefficient depends on the local motion of each end. This is illustrated in Fig. 11(b), where the friction at the back is due to the slip-bonds when the back end of the cell is retracting $\dot{x}_{b}>0$ and $\Theta\left(\dot{x}_{b}\right)=1$.

Note that the force that pulls on the adhesions during retraction [in the exponential function in Eq. (28)] is not only given by the elastic spring force (as in the previous models presented above) but is countered by the local force of actin polymerization that is pushing the membrane.
Similarly, the dynamics of the adhesions are given by

$$
\dot{n}_{f / b}=r\left(1-n_{f / b}\right)-n_{f / b} \exp \left(\frac{-v_{f / b}+k\left(x_{f}-x_{b}-1\right)}{f_{s} n_{f / b}}\right) \text {. }
$$

The dynamics of the polymerization/tread-milling of the actin is now calculated separately on both sides of the cell, given by

$$
\dot{v}_{f / b}=-\delta\left(v_{f / b}-v_{f / b}^{*}\right),
$$

where

$$
v_{f / b}^{*}=\beta \frac{c_{s}}{c_{s}+\tilde{c}\left(x_{f / b}\right)} .
$$

The distribution of the inhibitor along the cell is affected by the competition between the treadmilling actin from both ends, so its given by Eq. (19) with: $v=v_{f}-v_{b}$.

\section{Results}

The solution of Eq. (31) is shown in Fig. 11(c). The solution shows that the cell remains unpolarized for $l<l_{c}$, and the actin tredmilling flows are equal at the two ends of the cell, given by $v_{f / b}=\beta(l /(c+l))$. A polarized cell for $l>l_{c}$ has a higher treadmilling flow from the front, and in the limit of large $l$ the treadmilling velocity approaches these limiting values: $v_{f}^{*}=\beta$ and $v_{b}^{*}=D / c$.

We find that there is a new length scale in the system, which determines the ability of the cell to elongate and reach polarization. For the unpolarized cell, we equate the protrusive forces at both ends to the restoring force of the spring

$$
k(l-1)=\beta\left(\frac{l}{c+l}\right),
$$

which yields the polarization length

$$
l_{p}=\frac{1}{2}(1-c)+\frac{\beta}{2 k}+\sqrt{c+\left(\frac{1}{2}(c-1)-\frac{\beta}{2 k}\right)^{2}} .
$$

By equating the critical length $l_{c}$ (22) to the polarization length $l_{p}(33)$, we obtain a critical value of $\beta$, above which the cell polarizes [Figs. 12(a) and 12(b)]

$$
\begin{aligned}
\beta_{c}= & \frac{d}{2 c}+c k+\frac{c^{2} k^{2}}{2 d} \\
& +\left(\frac{c k-d}{2 c d}\right) \sqrt{d^{2}+2 c(1+2 c) d k+c^{2} k^{2}} .
\end{aligned}
$$

As for the previous model (Sec. II B), the interesting behavior is for a cell that has a rest length that is smaller than $l_{c}>1$. For such a system we demonstrate the dynamics for different values of $\beta$ (above and below $\beta_{c}$ ), in Figs. 12(d)-12(1). For $\beta<\beta_{c}$, the cell elongates symmetrically [Figs. 12(d)-12(f)], but does not polarize (no spontaneous symmetry breaking). For $\beta>\beta_{c}$, we find regimes of smooth motion $[\beta=8$, Figs. $12(\mathrm{~g})-12(\mathrm{i})]$ and stick slip $[\beta=11$, Figs. 12(j)-12(1)] for parameters that correspond to these phases in the phase diagram of the stationary treadmilling system (Fig. 2). Note that for any fixed value of $\beta$ Eq. (34) provides a critical value of the cell stiffness $k$, above which $\beta<\beta_{c}$ and the cell can not elongate over the critical length, so becomes immobile.

When the cell is within the stick-slip regime, it may end up after each slip event with a cell length that is shorter than 

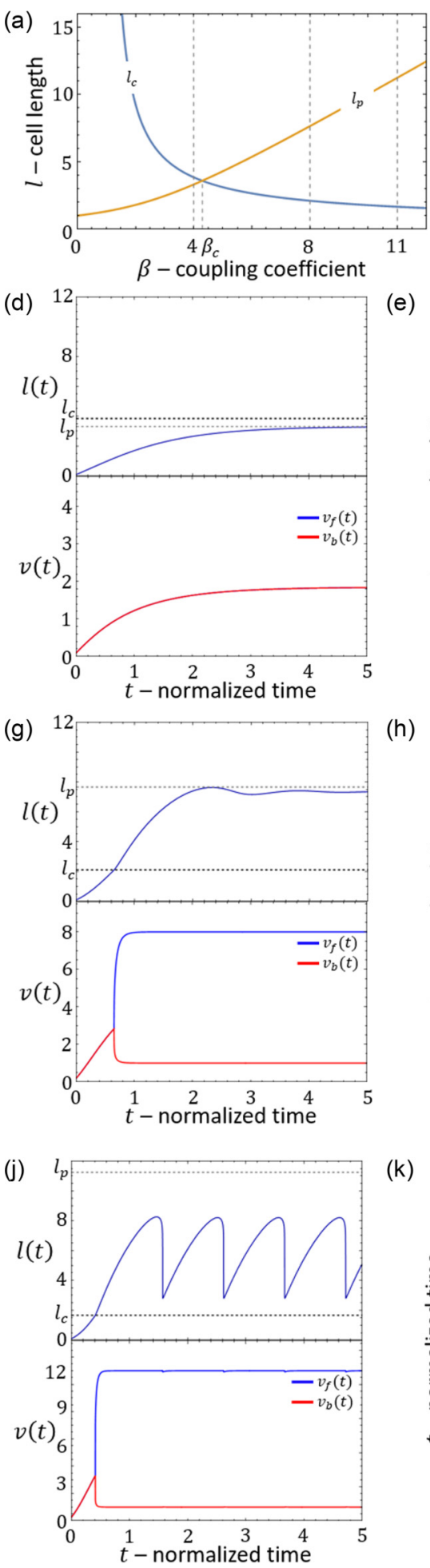

(h)

(k)
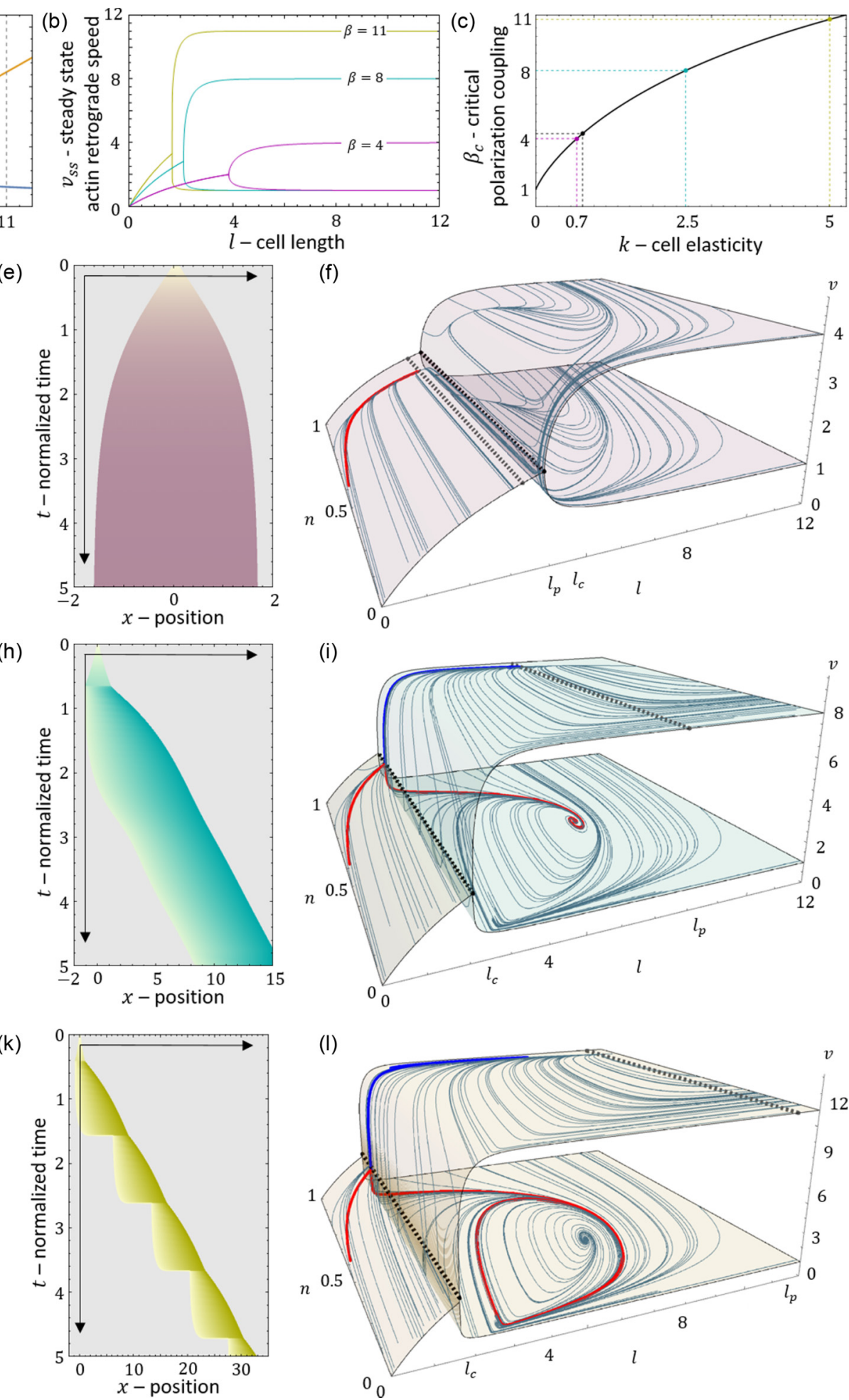

FIG. 12. The polarization length. (a) The critical length $l_{c}$ (blue) and polarization length $l_{p}$ (orange) as a function of the coupling coefficient $\beta$. Gray dashed lines indicate the sample points of $\beta=4,8$, and 11. (b) The steady-state velocity profile as a function of length for $\beta=$ 4,8 , and 11 (purple, teal, and yellow curves, respectively). (c) The critical coupling coefficient as a function of cell elasticity $k$. The purple, teal, and yellow dashed curves denote the critical values of $k$ for the values of $\beta$ used in (b) and (d)-(l). The black dashed line denotes the critical value of $\beta$ for $k=0.8$ [which is the value of $k$ used in (d)-(l)]. [(d)-(f)] Time series, kymograph, and phase space for $\beta=4$. [(g)-(i)] Time series, kymograph, and phase space for $\beta=8$. [(j)-(l)] Time series, kymograph, and phase space for $\beta=11$. In (d), (g), and (i), the upper blue curve is the time series of the cell length. Dashed black/gray lines are the critical/polarization lengths. Lower red/blue curves represent the time series of the actin retrograde flow speed at the front/back respectively. In (e), (h), (k), the purple/teal/yellow colors represent $\beta=4,8$, and 11. In (f), (i), and (1), the red/blue curves represent the trajectories at the $l-n_{f}-v_{f} / l-n_{b}-v_{b}$ phase spaces (upper/lower part of the surface). Parameters: $\kappa=20, f_{s}=5, r=5, k=0.8, c=3.85, \delta=250$, and $D=3.85$. 

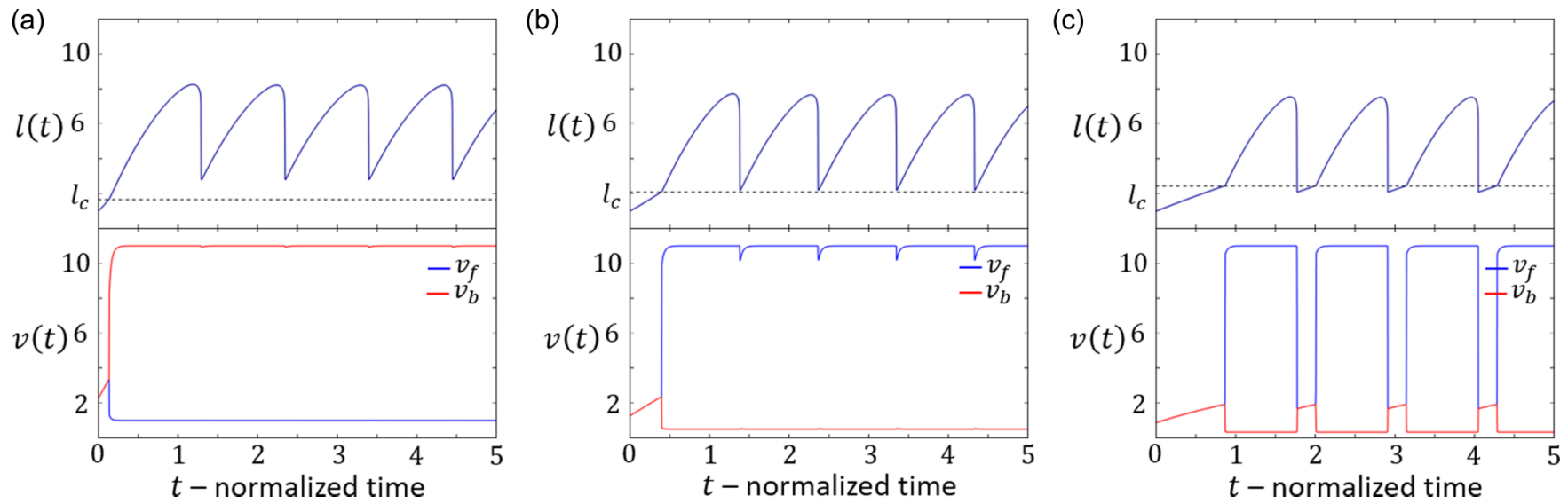

FIG. 13. Time series of the cell length (dark top panels) and the retrograde flow speed (bottom) of the symmetric system for different values of the parameter $c$ : (a) $c=3.85$, (b) 7.7, and (c) 11.55. Black dashed line in the upper panels is the critical length of polarization $l_{c}$. Red/blue curves in the lower panels represent the actin flow at the front/back. Other parameters: $\beta=11, D=3.85, f s=5, \kappa=20, r=$ $5, k=0.8$, and $\delta=250$.

$l_{c}$. Since in the symmetric model the protrusive force acts locally at both ends, the cell elongates symmetrically until $l>l_{c}$ and it repolarizes. This is demonstrated in Fig. 11(b). In Fig. 13, we show the effect of the parameter $c$ on the stick-slip migration, where increasing $c$ increases $l_{c}$ [Eq. (22)] and increases the time it takes the cell to repolarize following each slip event.

Furthermore, since the cell is effectively stalled when its length is shorter than $l_{c}$, the time it takes for the cell to expand back to $l_{c}$ after each stick-slip event determines the overall migration velocity. This is demonstrated in Figs. 14 and 15 .

During each stick-slip event, since the cell loses polarity, it may repolarize in a new direction. This occurs when we add random noise to the equations of motion: the system is solved as a stochastic differential equation where additive noise is added to the actin flow $v_{f}$ and $v_{b}$. For different amplitudes of the noise term, we plot in Fig. 16(a) the probability $p$ that the cell changes its direction of motion per stick-slip event. We find that for each level of noise there is a critical value (a)

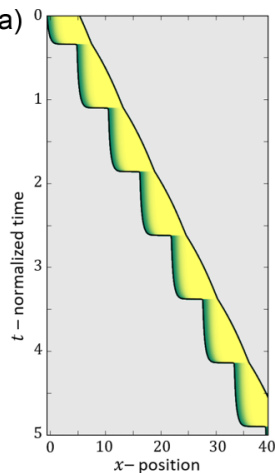

(d)

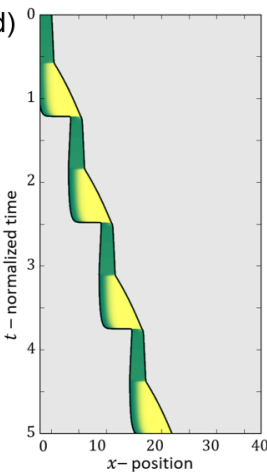

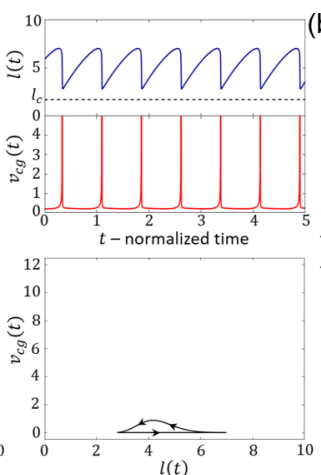
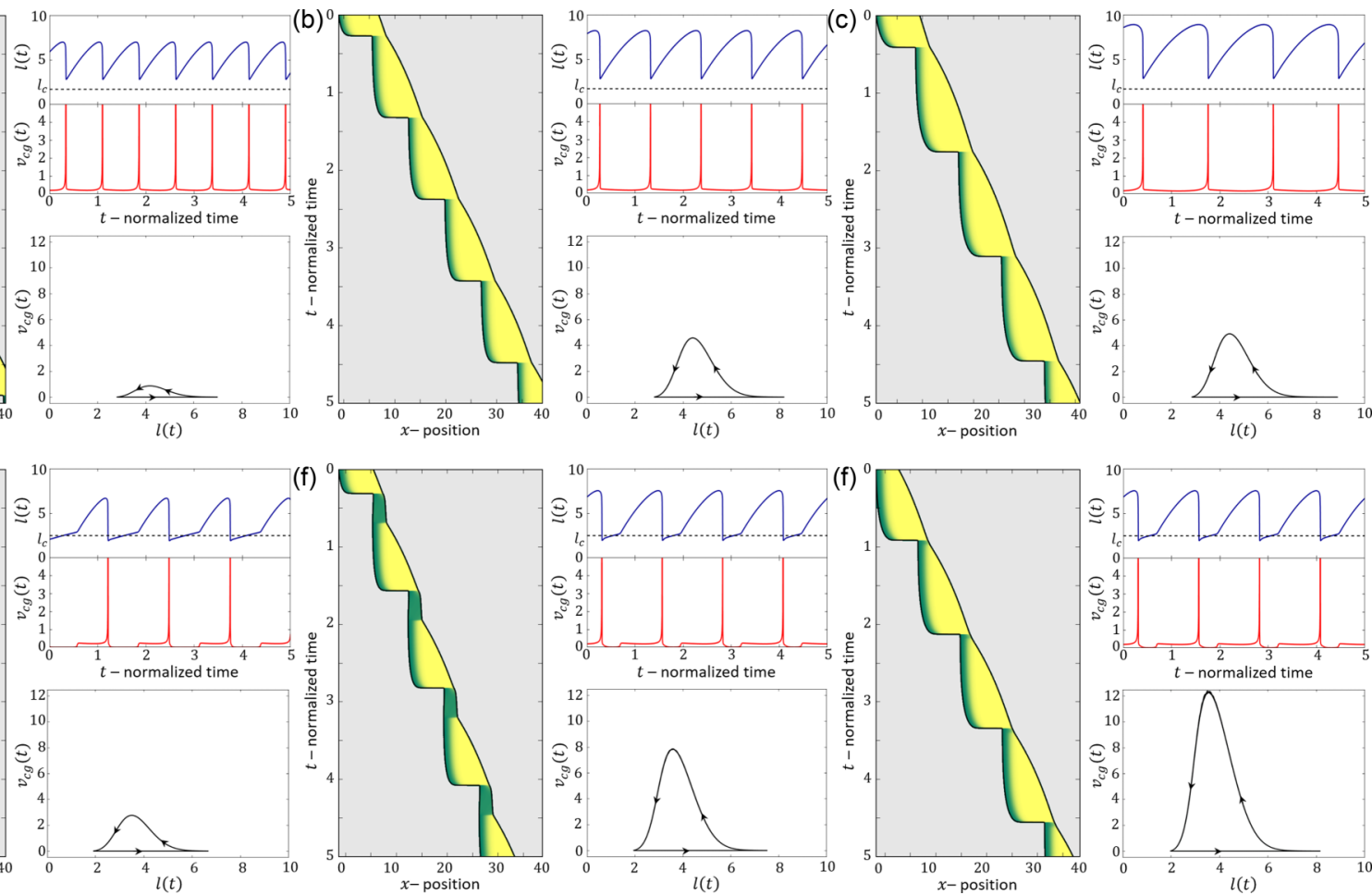

FIG. 14. The effect of $l_{c}$ on the overall velocity of migration during stick slip: [(a)-(c)] Above the critical length. $c=3.95, r=$ 3, 5, and 7. (Left) kymograph, (right top) cell length and speed time series. (Bottom) phase space. Parameters: $\beta=11, D=3.95, k=$ $0.8, f_{s}=5, \kappa=20, \delta=210$. [(d)-(e)] Below the critical length. $c=11.85, r=3,5$, and 7. (Left) kymograph, (right top) cell length and speed time series. (Bottom) Phase space. Parameters: $\beta=11, D=3.95, k=0.8, f_{s}=5, \kappa=20$, and $\delta=210$. 
(a)

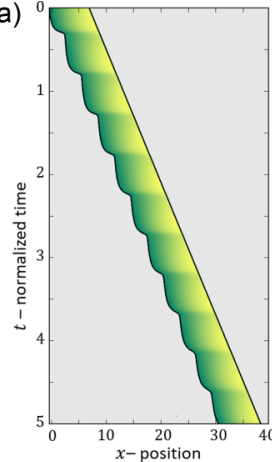

(d)

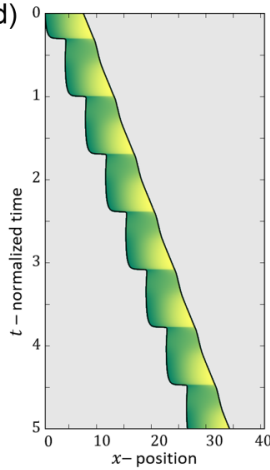

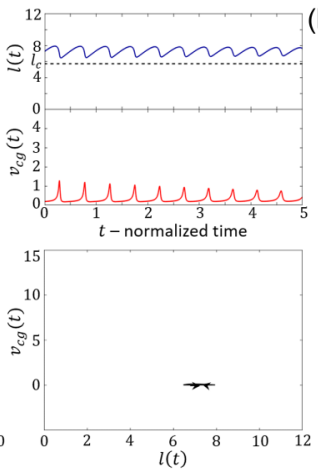

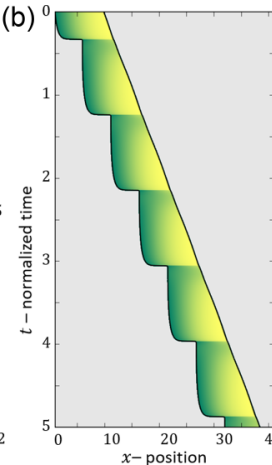

$(e)$
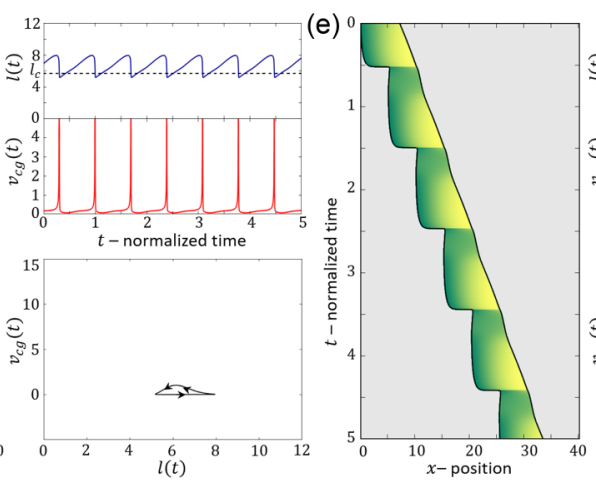
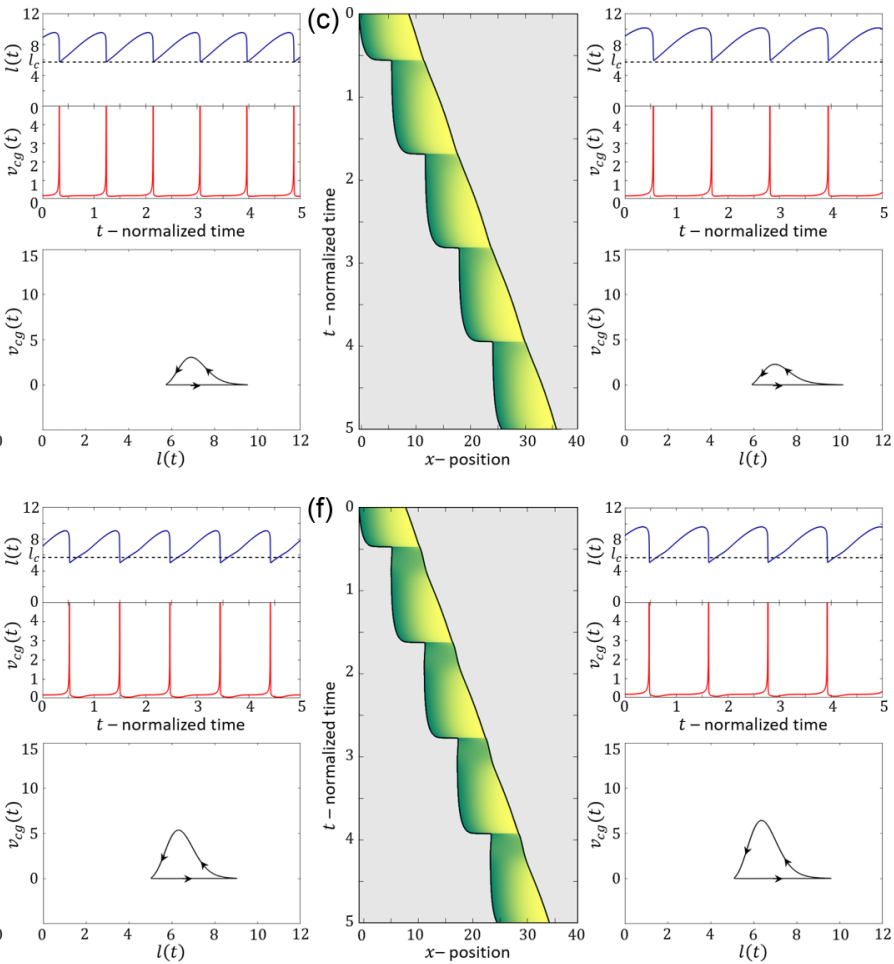

FIG. 15. Effect of $l_{c}$ on the overall migration velocity during stick-clip motion. [(a)-(c)] Cell length is above the critical length. $c=5, r=$ 2, 4, and 6. (Left) Kymograph, (right top) cell length and speed time series. (Bottom) phase space. Parameters: $\beta=11, D=3.95, k=$ $0.8, f_{s}=5, \kappa=20$, and $\delta=210$. [(d)-(e)] Below the critical length. $c=6, r=2,4$, and 6. (Left) Kymograph, (right top) cell length and speed time series. (Bottom) phase space. Parameters: $\beta=14, D=20, k=1, f_{s}=5, \kappa=20$, and $\delta=120$.

of the actin response rate $\delta$ [Eq. (30)] that sets a threshold between $p=0$ and 0.5 . During each stick-slip event, when $l<l_{c}$, the actin flow velocities on both sides approach each other exponentially with a timescale of $\delta^{-1}$. As $v_{f / b}$ approach within the noise amplitude, they cross each other [Figs. 16(b) and 16(c)], and the side that has the higher flow when $l=l_{c}$ determines the new direction of cell motion. The dynamics around the critical $\delta$ where the turning probability increases strongly, are demonstrated in Fig. 17.

\section{COMPARISON TO EXPERIMENTS}

When comparing the results of our fully symmetric model to the experimental observations of motile cells along onedimensional tracks, we need to note that the cellular system is very noisy. Nevertheless, our model exposes distinct motility patterns that should underlie the noisy motion of the cells.

First, we wish to show that the main classes of cell behaviors, which is observed within a uniform population of identical cells (Fig. 18) can be qualitatively spanned by the model if, for example, the actin treadmilling activity (model parameter $\beta$ ) is variable within the population.

Next, we focus on the stick-slip migration pattern, and compare several examples of such observed cell trajectories to the dynamics predicted by the model (Fig. 19). We find that there is strong similarity between the dynamics exhibited by the cell and in the model, regarding the oscillations in the cell length and velocity. The limit-cycle trajectory predicted by the model can be observed in the experimental data. For slightly different set of parameters, we find that the model gives rise to other stick-slip regimes observed in the experiment: a cell with low-frequency of small-amplitude stick-sip events (Fig. 20), and a cell with high-frequency of large-amplitude stick-slip events (Fig. 21).

In Fig. 22, we give a wide range of different cell migrations observed in experiments, demonstrating that the model can produce a similar range of cell migration patterns. In Fig. 22(a), we show a cell that seems to be oscillating between being polarized to the right and to the left, such that it hardly migrates over time. In our model, a similar behavior may occur if the cell is in the stick-slip regime, and following each slip event it is shorter than the critical length, allowing for noise to induce direction changes (as explained in Figs. 16 and 17).

In Fig. 22(b), a kymograph is shown of a cell that is initially at rest, and elongates symmetrically. At some later time it breaks the right-left symmetry and moves in a persistent manner. This behavior is well described by our model, where the rest-length of the cell is below the polarization length, but the conditions are such that $\beta>\beta_{c}$ and it elongates symmetrically until it reaches a length that is longer than the critical length $l_{c}$. At a later time point we decreased the value of the surface adhesion strength $r$, leading to a transition from long-slow to short-fast migration mode, which seems to fit this cellular behavior (similar to Fig. 6).

In Fig. 22(c), we plot a kymograph of a cell that is persistently moving on a 1D track in the stick-slip regime. During each stick-slip event the progression of the cell's leading edge is slightly modulated, while at the back there are periodic extensions of lamellipodia that undergo large retractions. This 
(a)

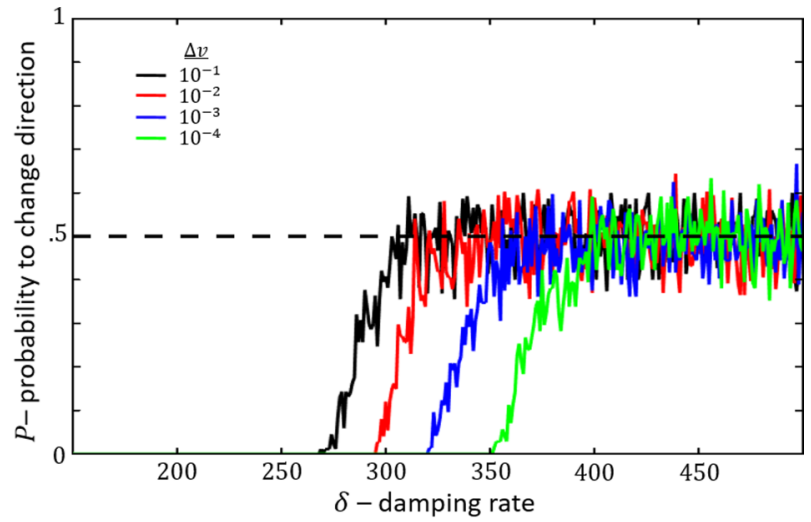

(b)

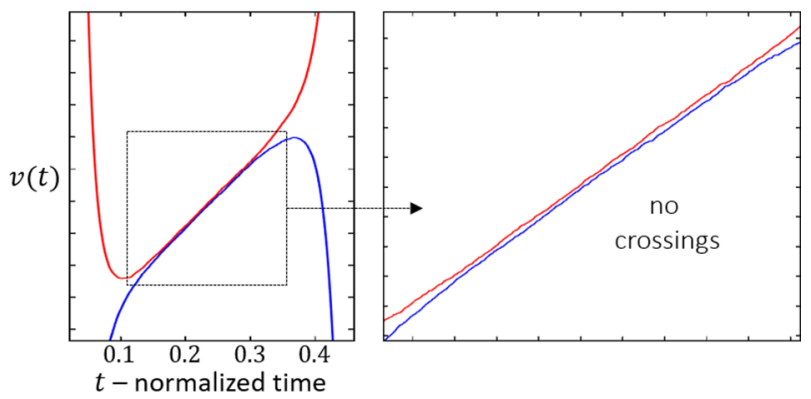

(c)

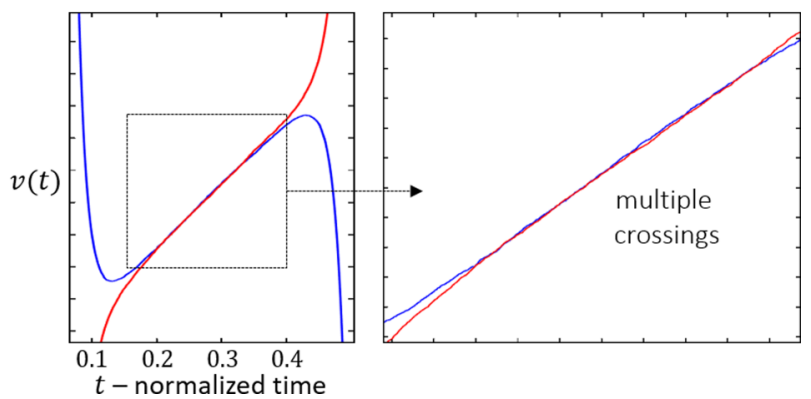

FIG. 16. (a) The probability to change direction as a function of the damping rate for different levels of noise in the actin velocity. (b) Demonstration of a stick slip event at which the noise level is low $\left(\Delta v=10^{-3}\right)$, such that $v_{f}$ (blue) and $v_{b}$ (red) do not cross when $l<l_{c}$, and the cell remains polarized. (c) Demonstration of a stick slip event at which the noise amplitude is sufficiently high $(\Delta v=$ $10^{-2}$ ), such that $v_{f}$ (blue) and $v_{b}$ (red) cross when $l<l_{c}$, which enable the polarization mechanism to switch directions. Parameters: $\kappa=20, f_{s}=5, r=5, k=0.8, c=11.55, D=3.85, \beta=$ 11 , and $\delta=300$.

behavior corresponds very well to the stick-slip behavior in our model.

Finally, in Fig. 22(d), we present a kymograph of a cell that is first observed to be migrating by slow stick-slip events. At some point the cell stops and rounds up, as it enters mitosis, from which two daughter cells emerge, both migrating with similar speeds, but one seems to be moving smoothly while the other exhibits some stick-slip cycles. We demonstrate a similar chain of migration changes using our model, by modulating the parameters that determine the actin treadmilling activity $(\beta)$ and adhesion strength $(r)$.

These comparisons serve to show that the model can describe the complex migration patterns of cells. The parameters used in the model may correspond to a unique migration mode, or correspond to regimes where different migration modes coexist. The transitions in the cell behavior from one migration mode to another may therefore correspond to the dynamics of the internal parameters of the cell. In addition, noise in the dynamical variables (such as in the actin treadmilling speed), can drive the enhanced polarization changes observed during stick-slip motion.

\section{CONCLUSION}

Cell motility involves a large number of cellular components, connected by a complex network of interactions. In addition, the system is noisy, which makes the analysis of cell migration a daunting task. In the present work, we developed a theoretical model aimed at exposing the motility patterns of cells moving along a one-dimensional track. One dimensional motion is both simpler to analyze experimentally, and describe theoretically, as well as being highly relevant to cells migrating within tissues during development $[17,18]$ and cancer progression $[16,19]$. It is also considered to be closely related to the migration of cells in many types of three-dimensional environments [36,37].

We find that the coupling between the cell length and the slip-bonds of adhesion molecules, through the springlike elasticity of the cell, provides the basic mechanism of stick-slip during cell migration. Furthermore, we show that when cells migrate and their polarization is maintained by the overall actin treadmilling flow, the cell length couples strongly to the polarization state of the cell: cells below a critical length do not polarize. While persistent smooth and stick-slip migration modes arise naturally in our model, we predict that stick-slip events may allow for noise-induced direction changes, as the cell recoils to less than the critical length at each stick-slip cycle.

The minimal model that we present recovers the rich variety of cellular migration patterns, which we then compare to experiments on migrating cancer cells (glioma). These comparisons validate the model and demonstrate how it can provide the framework for understanding the complex migration patterns exhibited by migrating cells.

Our model makes detailed predictions regarding the dependence of the cellular migration modes on the coarse-grained parameters of the model, which describe the cell's mechanics $(k)$, actin-polymerization activity $(\beta)$ and surface adhesion $(r)$. We predict a reentrant smooth migration regime as function of the surface adhesion, with stick-slip occurring only at the intermediate regime (Figs. 2, 8, and 10).

Despite the fact that the motile behavior of cells is highly dominated by noise, our model helps to expose the underlying deterministic patterns of motility [27] that drive the cellular motion, which may be partially masked by noise. We show that the noise can also drive dramatic transitions between the different motility patterns, since the cell may reside close to the transition lines, or in a regime of coexistence, between such modes. These results offer a new explanation for the large "phenotypic heterogeneity" (CCV) that is observed in cell migration experiments, even under well controlled conditions and monoclonal cell population. These results offer a new framework to explain experimental observations of migrating cells, resulting from noisy switching between un- 

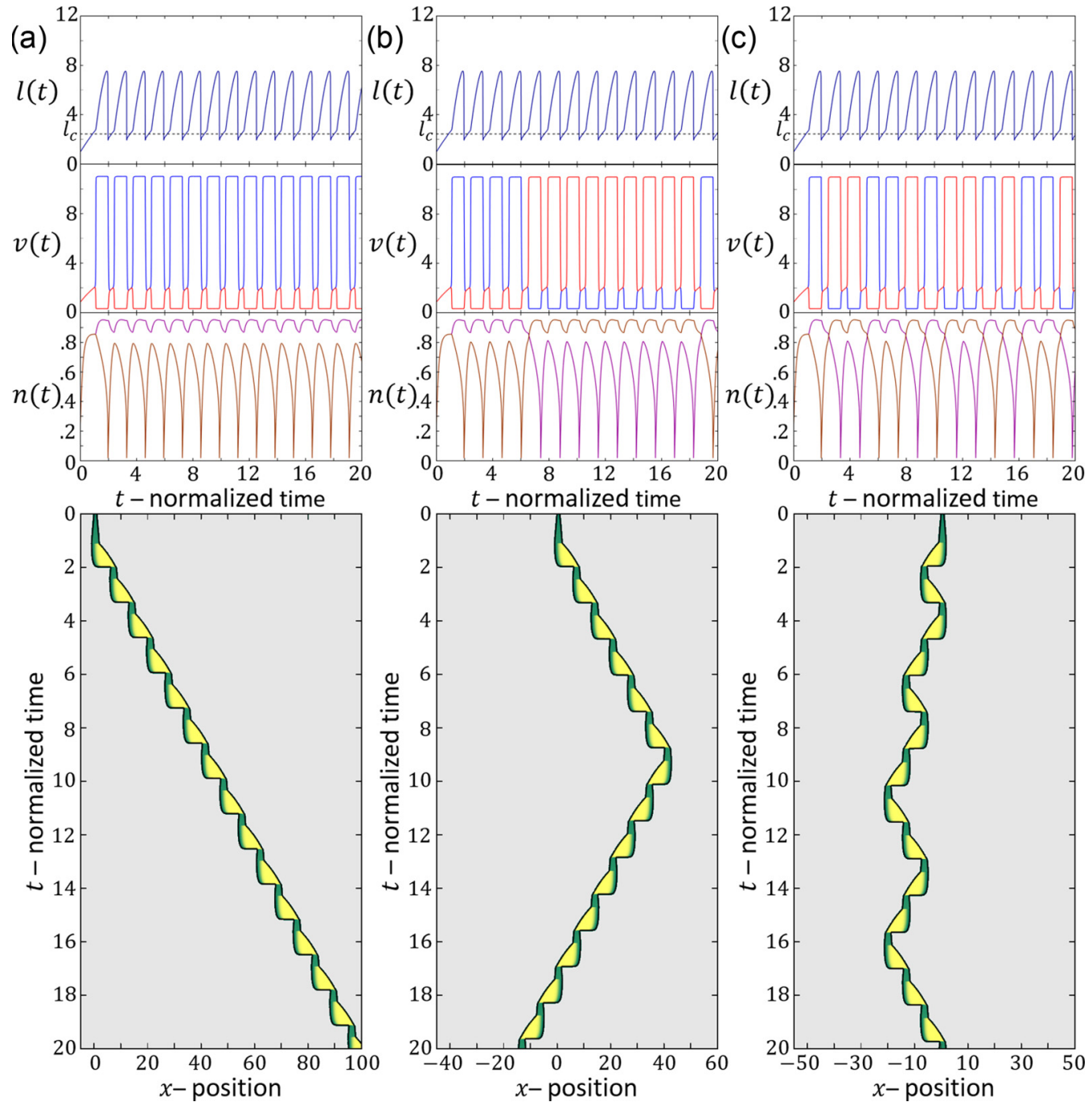

FIG. 17. Demonstration of direction changes events for different values of $\delta$ with noise amplitude of $\Delta v=10^{-3}$ [see Fig. 16(a)]. Upper/middle/lower panels correspond to the time series of the cell length/actin flow/adhesion concentration for $\delta=300,325$, and 350, (a)-(c) from left to right respectively. At the bottom, there are the corresponding kymographs. Parameters: $\kappa=20, f_{s}=5, r=5, k=$ $0.8, c=11.55, \beta=11$, and $D=3.85$.

derlying deterministic migration modes. Small fluctuations in the cellular components that are greatly amplified due to shifting the cells' internal state between coexisting migration modes, and across phase transition lines. The rich- ness in migration modes that our model predicts for cells with identical or slightly different internal states offers a new paradigm to explain phenotypic heterogeneity in cell migration.

(a)
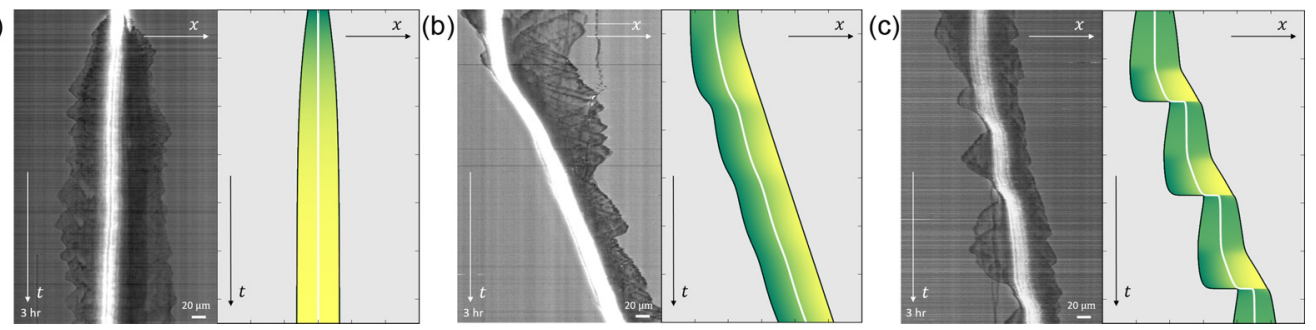

FIG. 18. Demonstrating the different migration modes as a function of the strength of the actin flow $(\beta)$, in comparison to observed cell migrations (C6 glioma cells seeded on laminin-coated lines of $5 \mu \mathrm{m}$ width, imaged every $30 \mathrm{sec}$ ). Kymographs correspond to total time of 3 hours: (a) a cell spreading symmetrically and not polarizing: $\beta=8.5$ (in our model $\beta<\beta_{c}$, Fig. 12). (b) Smooth migration: $\beta=9$. Note that the length variations at the front are associated with actin waves which propagate in the cells and are not part of this model [15]. (c) Stick-slip migration: $\beta=10.5$. Other model parameters: $\kappa=20, f_{s}=5, r=3, k=0.75, c=7.12$, and $D=15.4$. The white line in the model kymographs denotes the trajectory of the cell's geometric center. 
(a)
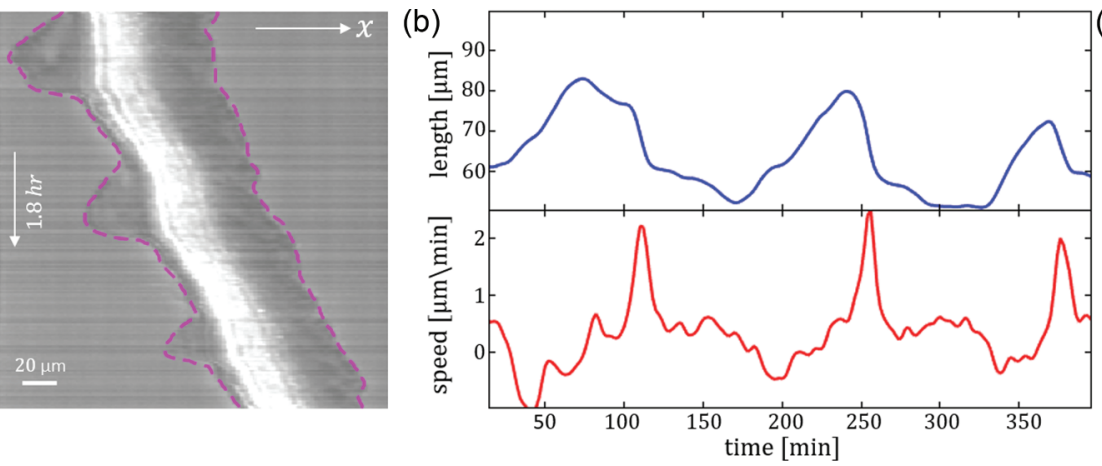

(e)

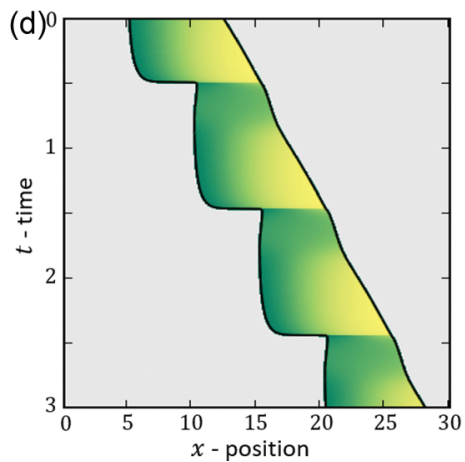

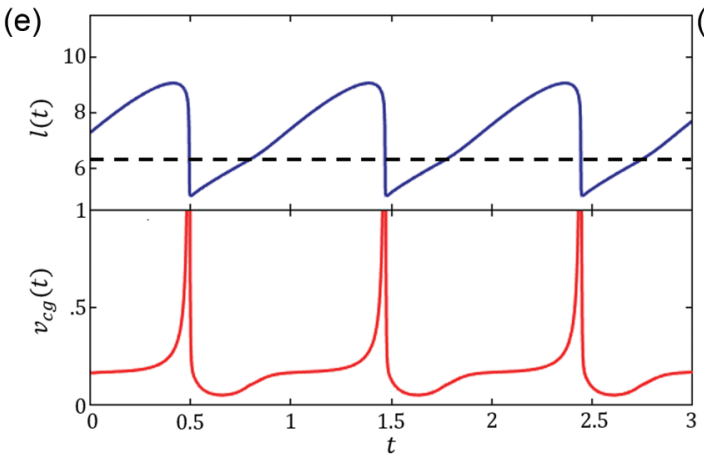
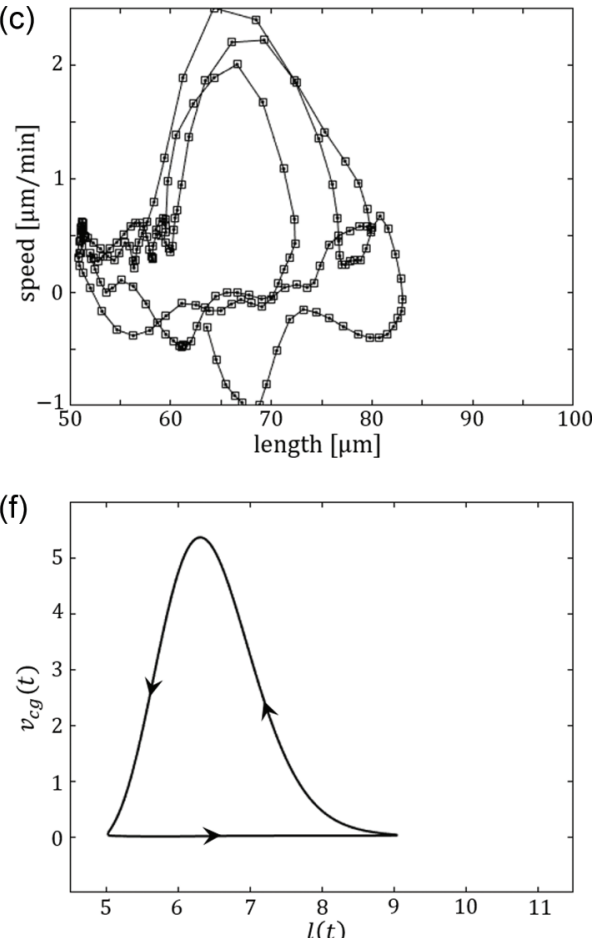

FIG. 19. Stick-slip migration analysis in experiments and the model. [(a)-(c)] Experiment: C6 glioma cells seeded on laminin-coated lines of $5 \mu \mathrm{m}$ width (imaged every $30 \mathrm{sec}$ ). Kymograph correspond to total time of 3 hours. (a) Kymograph of a cell with stick-slip migration. (b) Upper panel is the time-dependent length of the cell. Lower panel is the cell speed (from the trajectory of the geometric center). (c) Phasespace trajectory of the cell. [(d)-(f)] Model results for a cell in the stick-slip regime: (a) kymograph, (b) length and velocity time series, and (c) phase space trajectory. parameters: $\beta=14, c=6, D=20, r=4, k=0.8, f_{s}=5, \kappa=20$, and $\delta=120$.

(a)

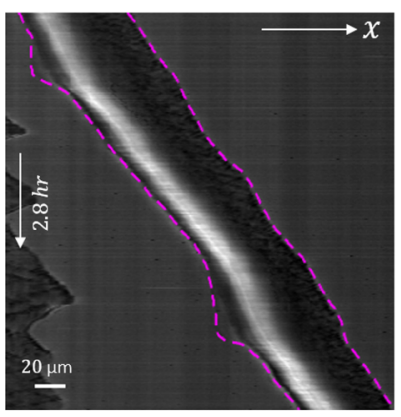

(d)

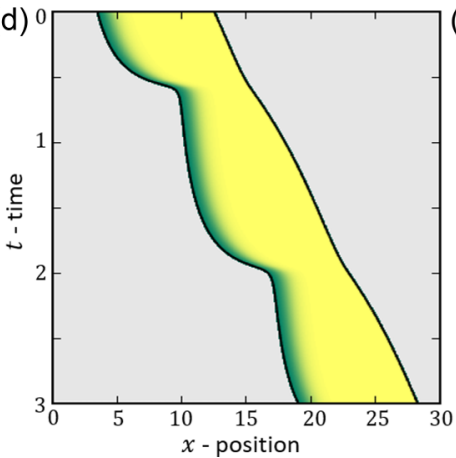

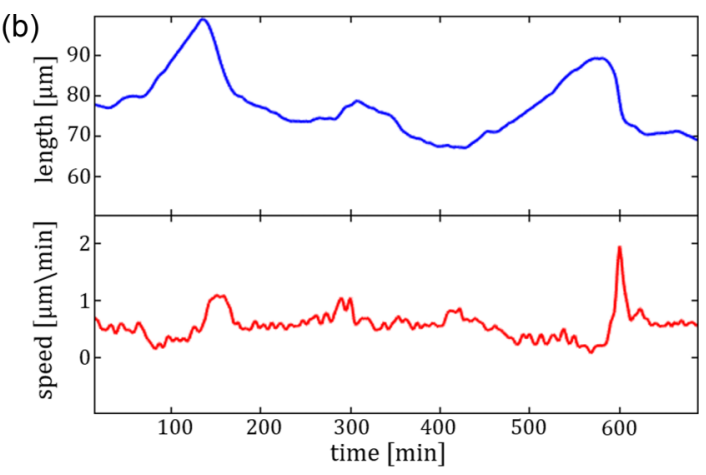

(e)

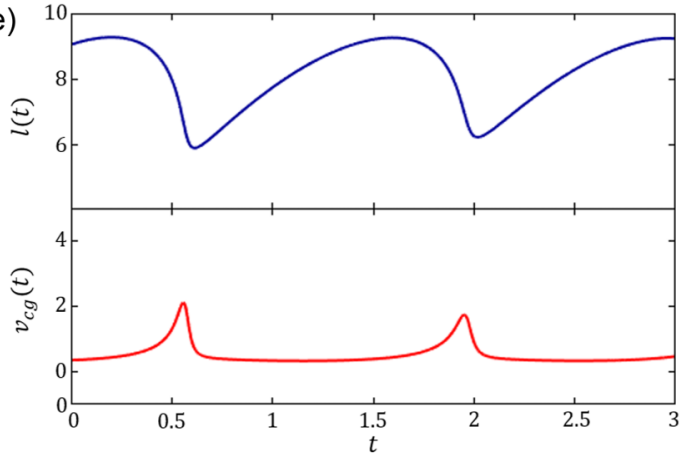

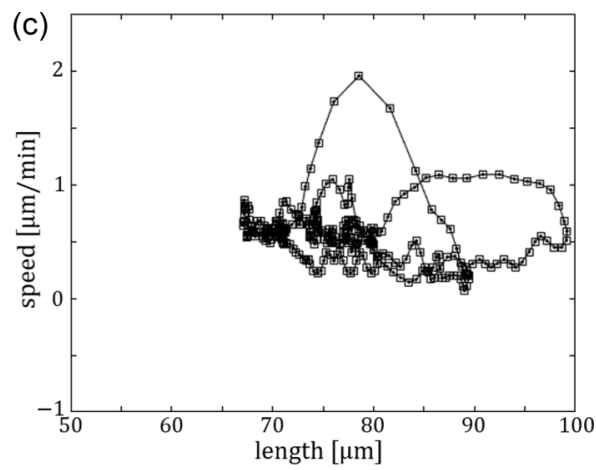

(c)

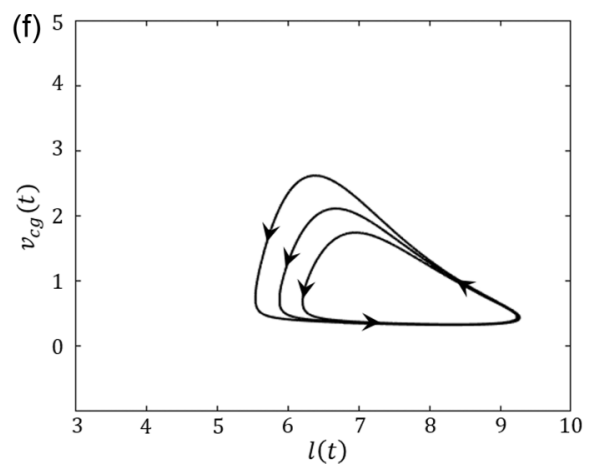

FIG. 20. Stick-slip migration comparison to experiment of C6 glioma cells migrating on laminin-coated lines of $5 \mu \mathrm{m}$ width (frames are $30 \mathrm{sec}$ apart). [(a)-(c)] Experiment. [(d)-(f)] Model calculation. For both we plot the kymograph, cell length and speed time series, and phase space trajectories. Model parameters: $\beta=12, c=8.37, D=10.86, k=0.9, f_{s}=5, r=6, \kappa=16$, and $\delta=150$. 
(a)
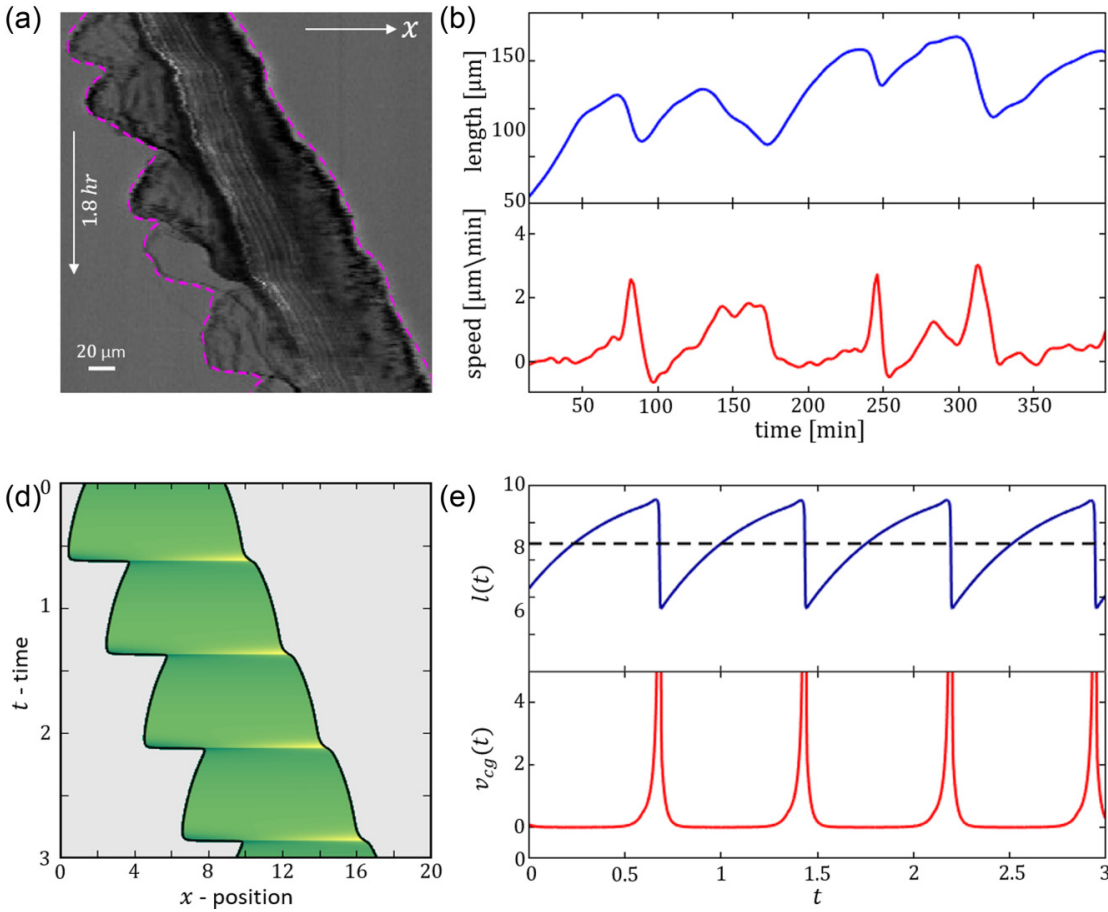

(e)

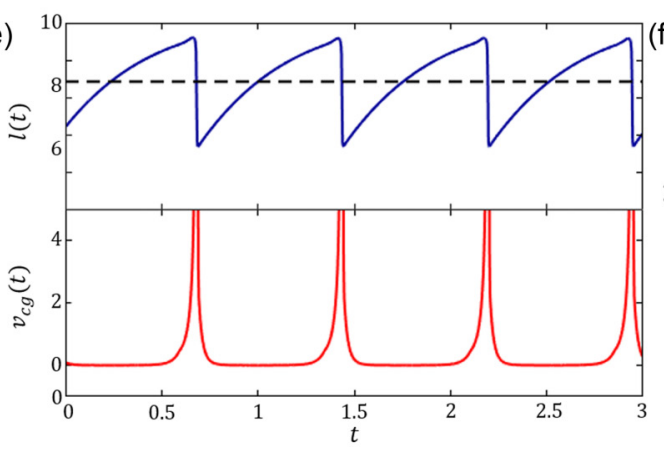

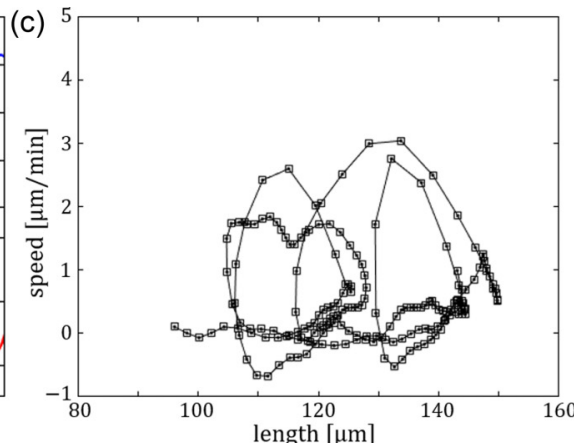

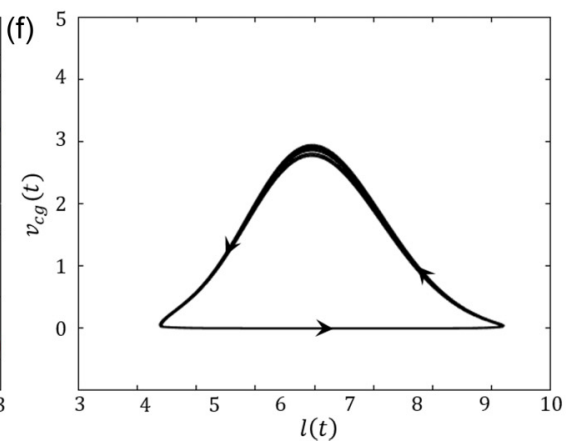

FIG. 21. As in Fig. 20. [(a)-(c)] Experiment. [(d)-(f)] Model calculation. Model parameters: $\beta=19, c=9, D=40, k=1.1, f_{s}=$ $5, r=0.4, \kappa=20$, and $\delta=120$.

We note that in the future our model could be extended to include more details of the cellular dynamics, such as stickslip of the leading edge, as well as explored in the context of cells moving in two [38] and three dimensions. Direct biochemical feedback between the actin flow or the myosin-II (polarity cue) and the adhesion [39], may also be considered.

Our results explain naturally many observations of cells that grow symmetrically, and migrate along $1 \mathrm{D}$ tracks. In addition, the model makes predictions that can motivate future experimental exploration. Finally, having a model for singlecell motility can serve as the basis for the description of collective cell migration [40], which goes beyond treatments of the cells as simplified self-propelled particles.

\section{ACKNOWLEDGMENTS}

We thank Pierre Sens for useful discussions. N.S.G. acknowledges that this work is made possible through the historic generosity of the Perlman family. N.S.G. is the incumbent of the Lee and William Abramowitz Professorial Chair of Biophysics and this research was supported by the Israel Science Foundation (Grant No. 1459/17). N.C.G. and P.M. acknowledge support by IFOM starting package, and the AIRC Foundation for Cancer Research in Italy (AIRC), Investigator Grant (IG) 20716 to N.C.G.

\section{APPENDIX A: PARAMETER VALUES ESTIMATION}

We give here a detailed discussion of the parameters values that are used in the model throughout the paper. Note that not all of these parameters have been directly measured, and therefore some remain as estimates.
(1) The traction force is obtained by traction force microscopy measurements [41]

$$
F_{\text {traction }} \sim 10[\mathrm{nN}]=10\left[\frac{\mathrm{kg} \mathrm{m}}{\mathrm{s}^{2}}\right] .
$$

In the model, the traction forces produced by the drag or sliding actin filaments are proportional to the actin flow

$$
F_{\text {traction }} \sim \alpha v \sim \gamma v .
$$

The velocity of the actin retrograde flow in glioma cells is obtained by direct measurment

$$
v=110\left[\frac{\mathrm{nm}}{\mathrm{s}}\right] \text {. }
$$

Therefore the friction coefficients can be estimated by

$$
\alpha, \gamma=0.09\left[\frac{\mathrm{kg}}{\mathrm{s}}\right] \approx 0.1\left[\frac{\mathrm{kg}}{\mathrm{s}}\right] .
$$

Throughout the model the friction coefficients are normalized in dimensionless units: $\alpha, \gamma=1$ [a.u], therefore

$$
1\left[\frac{\mathrm{kg}}{\mathrm{s}}\right] \rightarrow 10 \text { [a.u]. }
$$

(2) Length and timescales.

The length scale, the cell's rest length $x_{0}$, is estimated through the kymographs by

$$
x_{0} \approx 10-100[\mu \mathrm{m}] \text {. }
$$

The timescale of the basal off rate of the linkers (adhesions), can not be estimated directly, and is approximated after fixing the parameters of the UCSP polarity model, such that the cell is in the persistent motion regime [26]. The 
(a)

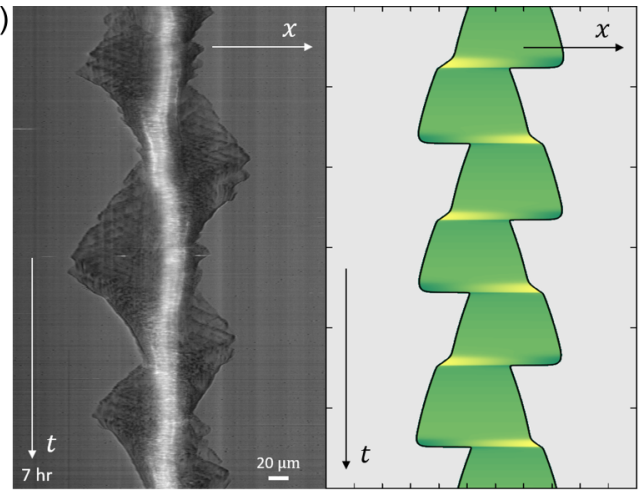

(c)

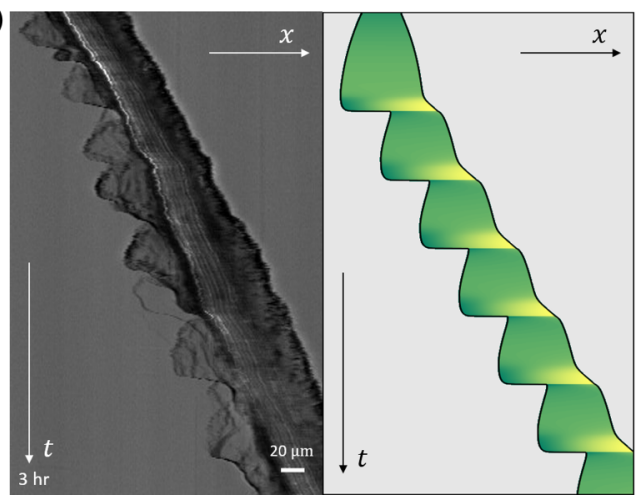

(b)

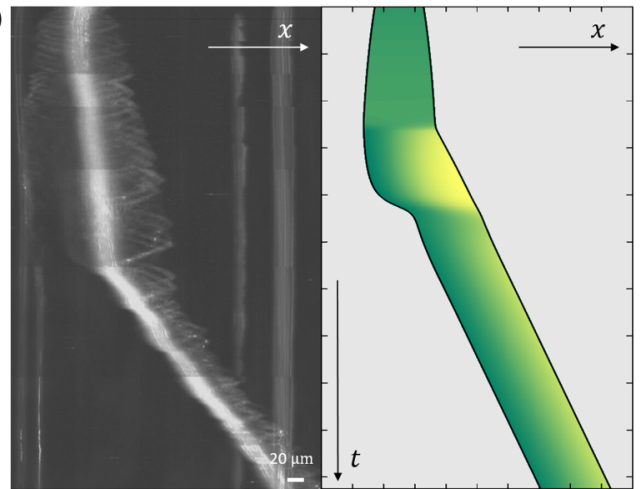

(d)

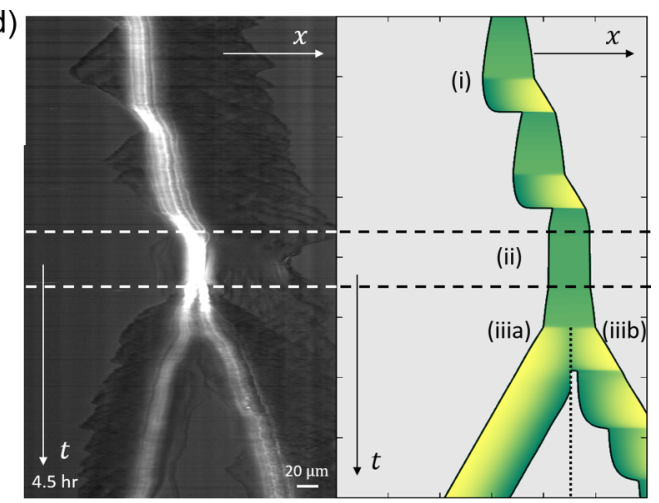

FIG. 22. Comparison of different migration patterns between experiments and model. [(a), (c), and (d)] Kymographs of C6 glioma cells migrating on laminin-coated lines of $5 \mu \mathrm{m}$ width (frames are $30 \mathrm{sec}$ apart). (a) Stick-slip motion with frequent direction changes. Model parameters: $\beta=18, c=9, D=30, k=1.05, f_{s}=5, r=0.5, \kappa=20$, and $\delta=220$. (b) Kymograph of 3T3 cells seeded on a nanofiber coated with fibronectin (image extracted from Ref. [15]). The cell initially spreads by developing lamellipodia in both directions, and eventually breaks symmetry and migrates persistently with an approximately constant length. Model parameters: $\beta=8, c=3.85, d=3.85, k=$ 2, $f_{s}=5, r=1, \kappa=20$, and $\delta=220$. (c) Persistent stick-slip motion, with large lamellipodia extensions at the rear of the cell (image extracted from Ref. [9]). Model parameters: $\beta=40, c=9, D=40, k=1.1, f_{s}=5, r=0.4 \kappa=20$, and $\delta=60$. (d) A cell performing stick-slip, goes into mitosis (nonmotile), divides and generates two daughter cells migrating in opposite directions. The transitions between the different stages are indicated by the horizontal dashed lines. Model parameters: (i) $\beta=40, r=10$; (ii) $\beta=15, r=10$; (iiia) $\beta=19, r=$ 12; and (iiib) $\beta=19, r=20$. Rest of the parameters $c=9, D=40, k=1.1, f_{s}=5, \kappa=20$, and $\delta=300$.

diffusion coefficient of different proteins in the cell [27], is estimated by

$$
D=1-10\left[\frac{\mu \mathrm{m}^{2}}{\mathrm{~s}}\right],
$$

which is normalized by

$$
\bar{D}=\frac{D}{x_{0}^{2} k_{\text {off }}^{0}} \rightarrow k_{\text {off }}^{0}=\frac{D}{\bar{D} x_{0}^{2}} .
$$

For the persistent regime, and throughout the analysis of the model, we choose $\bar{D}=4[\mathrm{a} . \mathrm{u}]$, therefore the rate $k_{\text {off }}^{0}$ can be estimated by

$$
k_{\mathrm{off}}^{0}=2.5 \times\left(10^{-4}-10^{-2}\right)\left[\mathrm{s}^{-1}\right] \approx 10^{-4}-10^{-2}\left[\mathrm{~s}^{-1}\right] .
$$

(3) UCSP parameters. The total concentration of the polarity cue per unit length $c_{\text {tot }}$, and the concentration at which the polarity cue saturates $c_{s}$ can not be estimated directly, and are chosen such that the ratio between the two fits a cell in the persistent regime (considering our previous choice of $D$ above)

$$
c=\frac{c_{\mathrm{tot}}}{c_{s} x_{0}}=4[\mathrm{a} . \mathrm{u}]
$$

The choice of the coupling $\beta$ can be estimated by

$$
\beta=\frac{v}{x_{0} k_{\text {off }}^{0}} \approx 0.1-10 \text { [a.u] }
$$

The rate $\delta$, at which the actin flow adjusts to changes in length, was previously estimated as $\delta \sim 0.033-0.1$ [s $\left.^{-1}\right][27]$, therefore,

$$
\left.\bar{\delta}=\frac{\delta}{k_{\text {off }}^{0}}=3.3-1000 \text { [a.u }\right] \approx 1-10^{3} \text { [a.u] } .
$$

(4) Cell mechanics parameters.

The elasticity of glioma cells, as measured by atomic force microscopy [41] is given by $K=700 \mathrm{~Pa}$. The normalized spring constant, which represents the effective cell elasticity can be approximated by multiplying $K$ by the height of the cell (estimated by $h=0.1 \mu \mathrm{m})$

$$
k=\frac{K h}{k_{\text {off }}^{0}}=7 \times\left(10^{-3}-10^{-2}\right)\left[\frac{\mathrm{kg}}{\mathrm{s}}\right]=\approx 0.01-1 \text { [a.u] }
$$

The total number of linkers is fixed as $N=50$ [42]. The susceptibility of the linkers $f_{s}$ is estimated by previous work by $2-5 \mathrm{pN}[24,42]$, therefore, the normalized susceptibility is 

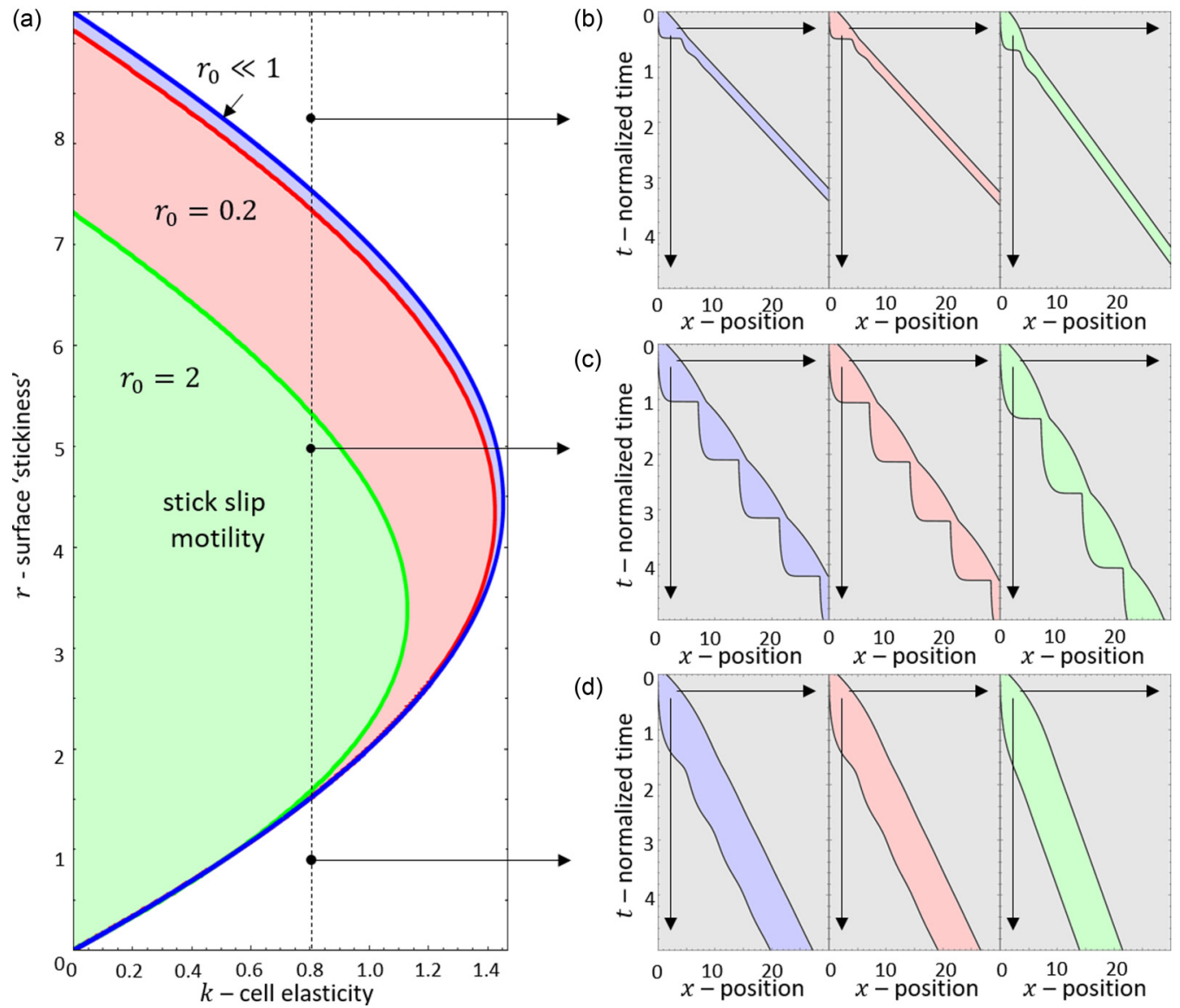

FIG. 23. The effects of the adhesion saturation parameter $r_{0}$ on the cellular dynamics. (a) $k-r$ phase diagram for different values of $r_{0}$. Blue/red/blue curves represent the transition between migration in constant length and stick slip for. [(a)-(d)] Kymographs of the model for $k=0.8$ and $r=2,5$, and 8.25. Blue/Red/Green colors represent $r_{0} \rightarrow 0 / r_{0}=0.2 / r_{0}=2$. Other parameters: $f_{s}=5$ and $\kappa=20$.

approximated by

$$
\overline{f_{s}}=\frac{f_{s} N}{x_{0} k_{\text {off }}^{0}}=2.5 \times\left(10^{-4}-0.1\right) \approx 10^{-3}-1[\mathrm{a} . \mathrm{u}] .
$$
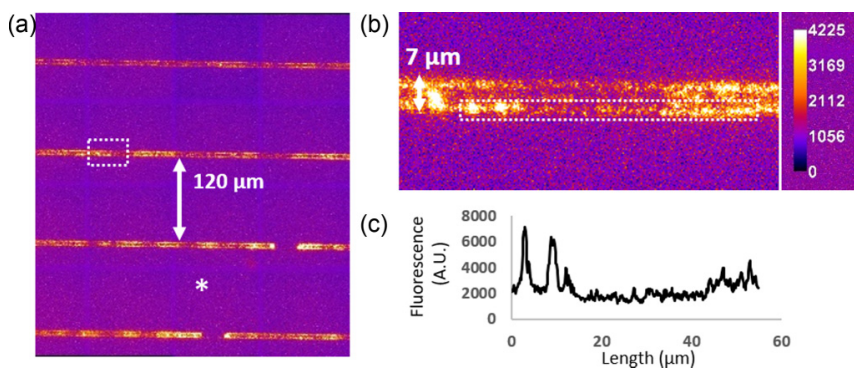

FIG. 24. Example of patterned laminin-coated linear tracks used in this study. The coating has been done with a mixture 1 to 5 with $2 \mathrm{~g} / \mathrm{ml}$ of alexa $647 \mathrm{BSA}$ and $10 \mathrm{~g} / \mathrm{ml}$ of laminin. (a) High resolution stitched field of view the robust patterning with occasional existence of several microns-scale gaps in coating (asterisk). (b) The zoomed image of the region marked by the dashed rectangle in (a), highlights the local submicrometer coating fluctuations, with variability in protein deposition highlighted by the color spectrum and quantified along a zone of $3 \mu \mathrm{m}$ in width, a size chosen to be in the range of usual focal adhesion size. (c) The fluctuations along the zone marked by the dashed rectangle in (b) are presented in the graph, illustrating local variation of intensity in protein concentration.
Throughout the analysis we choose $f_{s}=5$, which is in the order of magnitude of the higher bound. The stiffness of the adhesion linkers is estimated from previous work [42] as $0.8 \mathrm{pN} / \mathrm{nm}$, therefore the normalized linker stiffness can be approximated by

$$
\bar{\kappa}=\frac{\kappa N}{k_{\text {off }}^{0}}=4-400\left[\frac{\mathrm{kg}}{\mathrm{s}}\right] \approx 10-1000 \text { [a.u] } .
$$

The adhesion strength to the substrate $r$, is determined by the ratio between the basal off rate $k_{\text {off }}^{0}$ and the on rate $k_{\text {on }}$. Throughout the analysis we work in the range of $r=\frac{k_{\text {on }}}{k^{0}} \sim$ $1-10$ [a.u], which is is similar to previous works $[24,42]$.

\section{APPENDIX B: ANALYSIS OF THE STICK-SLIP PHASE DIAGRAM AS FUNCTION OF THE ADHESION SATURATION PARAMETER $\boldsymbol{r}_{0}$.}

In Eqs. (1) and (2), the parameters $\alpha$ and $\gamma$ should be multiplied by a factor of $r /\left(r+r_{0}\right)$, but throughout the main text (Fig. 2) we treated the limit of $r_{0} \rightarrow 0$. Here we show the effects of a finite value of $r_{0}$, where in Eqs. (15) and (16), we multiply $\alpha$ and $\gamma$ by $r /\left(r+r_{0}\right)$. Figure 23 demonstrates that as the value of $r_{0}$ increases the stick-slip region along the $k-r$ phase diagram decreases, and the cells migrate with a slower speed. 

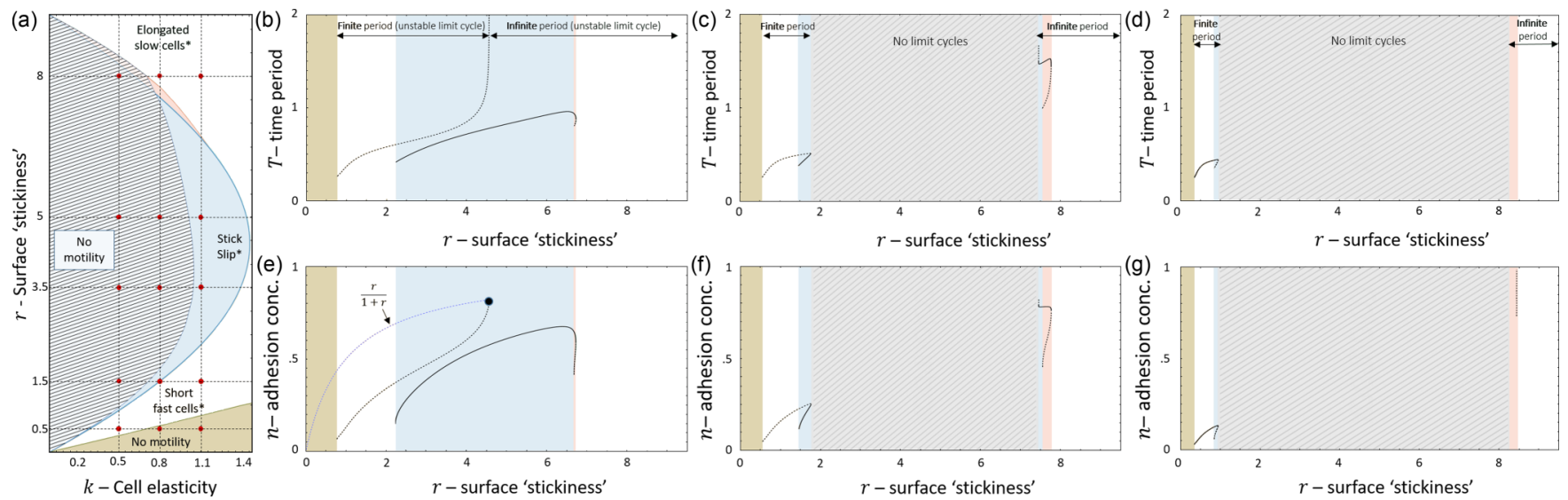

FIG. 25. (a) $k-r$ phase diagram as presented in Fig. 9. Black dashed lines correspond to the $r$ cross section for $k=0.5,0.8$, and 1.1 and $k$ cross sections of $r=0.5,1.5,3.5$, and 5, 8. Red dots correspond to intersections (dynamics at the intersections are shown in Figs. 26-28). [(b)-(d)] The time period of the limit cycles in the model along the cross sections of $k=1.1 / 0.8 / 0.5$ respectively. Black dashed line indicate the unstable limit cycles. Black solid lines indicate the stable limit cycles. [(e)-(g)] Maximal amplitude of the $n$ state variable as a function of $r$ for the limit cycles in the vector field along the cross section of $k=1.1 / 0.8 / 0.5$, respectively. Black dashed curves correspond to the unstable limit cycles. Solid black curves correspond to the stable limit cycles. Dashed blue curve corresponds to the $n$ value of the fixed point in the region where $l<x_{0}(l<1$ in the normalized units). Black dot corresponds to the transition between a finite and infinite period of the discontinuous unstable limit cycle (the $n$ cross section in which the unstable limit cycle crosses the section where $n=\frac{r}{1+r}$. Parameters: $f_{s}=5$, $\kappa=20, \beta=11, c=3.85$, and $D=3.85$.

\section{APPENDIX C: EXPERIMENTAL METHODS}

\section{Cell culture}

C6 rat glioma cells were obtained from ATCC and grown in High Glucose DMEM supplemented with $10 \%$ heat inactivated FBS (HI-FBS) and glutamine (Invitrogen). Graded brain tumor specimens were obtained with informed consent, as part of a study protocol approved by the SingHealth Centralised Institutional Review Board A (Singapore). From one of those specimen, derived human glioma propagating cells NNI21 (generous gift from Carol Tang laboratory, National Neuroscience Institutte Singapore were isolated and cultured as described previously [43] as tumor spheres in high glucose DMEM/F12 (1:1) supplemented with sodium pyruvate, nonessential amino acid, penicillin/streptomycin, glutamine, B27 supplement (Invitrogen), bFGF (20 ng/ml), EGF (20 ng/ml) (PeproTech), and heparin ( $5 \mathrm{~g} / \mathrm{ml})$ (Sigma). For transfection and migration assays, NNI21 were cultured as monolayers on laminin $(10 \mathrm{~g} / \mathrm{ml})$ coated petri dishes for 3-5 days before transfection. HGPCs transfections were performed with a Neon electroporator (Invitrogen) as per manufacturerâs recommendations using vinculin-mCherry (gift from P. Kanchanawong, Mechanobiology Institute, National University of Singapore, Singapore) as an adhesion rapporteur and GFP-Plasma Membrane (GFP-PM, Clonetech) for plasma membrane staining.

\section{Micropatterning}

$5 \mu \mathrm{m}$ lines were printed on glass coverslips using deep UV photopatterning technique as directed in [44]. Briefly, 25-mm glass coverslips were plasma-treated for $5 \mathrm{~min}$ and incubated for $1 \mathrm{~h}$ at room temperature (RT) with poly-llysineâgraftedâpolyethylene glycol $(0.1 \mathrm{mg} / \mathrm{ml}$, pLL-PEG, SuSoS) diluted in Hepes [10 mM (pH 7.4)]. After washing in water, the pLL-PEGâcovered coverslip was placed with the polymer brush facing downward onto the chrome side of a quartz photomask for photolithography treatment (7-min ultraviolet-light exposure). Subsequently, the coverslip was removed from the mask and coated with laminin $(10 \mu \mathrm{g} / \mathrm{ml})$ (Invitrogen) diluted in dPBS for $1 \mathrm{~h}$ at $37^{\circ} \mathrm{C}$. Cells were seeded on the patterns and incubated at $37^{\circ} \mathrm{C}$. Cell imaging typically started within the following hour.

\section{Microscopy}

Phase contrast of live specimens were performed on a Leica AM TIRF MC system equipped with temperature, humidity, and $\mathrm{CO} 2$ control. Long term imaging was done using a 10X objective (Leica HCX PL FLUOTAR 10x/0.30NA PH1 Objective). Acquisitions were typically obtained over a period varying from 2 to $12 \mathrm{~h}$ (1 image/30 s).

\section{APPENDIX D: CHARACTERIZATION OF THE HETEROGENEITY OF THE ADHESIVE COATING ON THE SUBSTRATE.}

In Fig. 24, we provide a direct measure of the heterogeneities in the amount of surface coverage by laminin along the one-dimensional stripes. These fluctuations occur spontaneously during the preparation of these stripes. In particular, there are high concentration puncta deposited along the track, as well as regions of overall high and low coverage that extend over tens of microns, i.e., over several cell lengths.

\section{APPENDIX E: CONCENTRATION PROFILE OF THE POLYMERIZATION INHIBITOR $c(x)$}

Consider a generic polarity cue which follows an advection diffusion transport along the length of the cell $l$. The polarity 

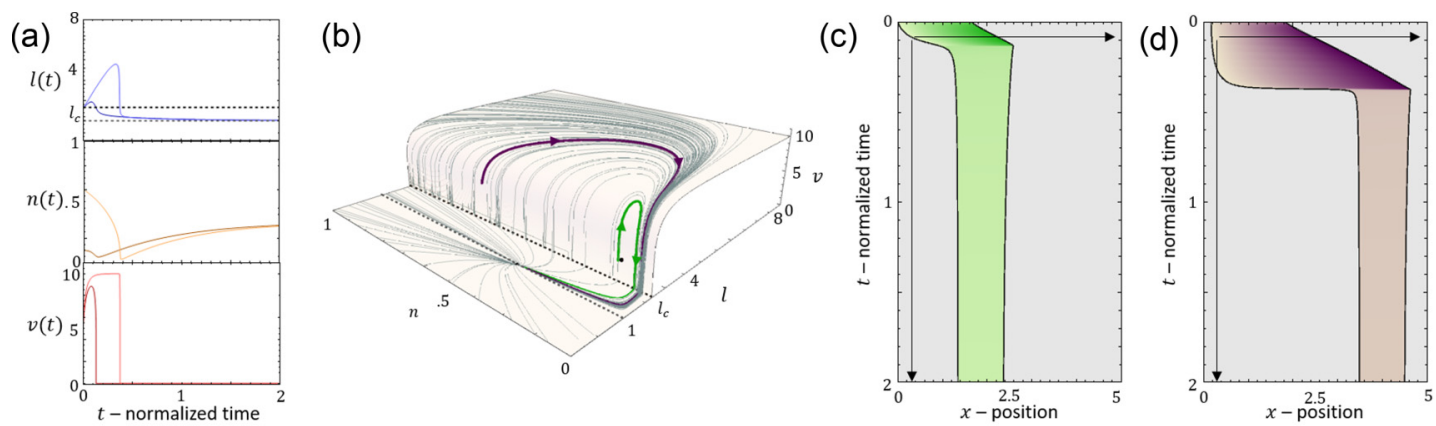

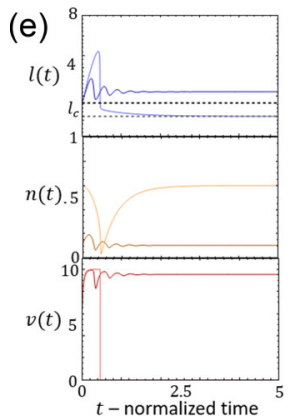

(f)

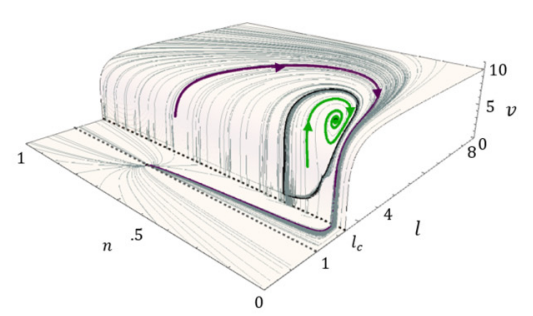

(j)
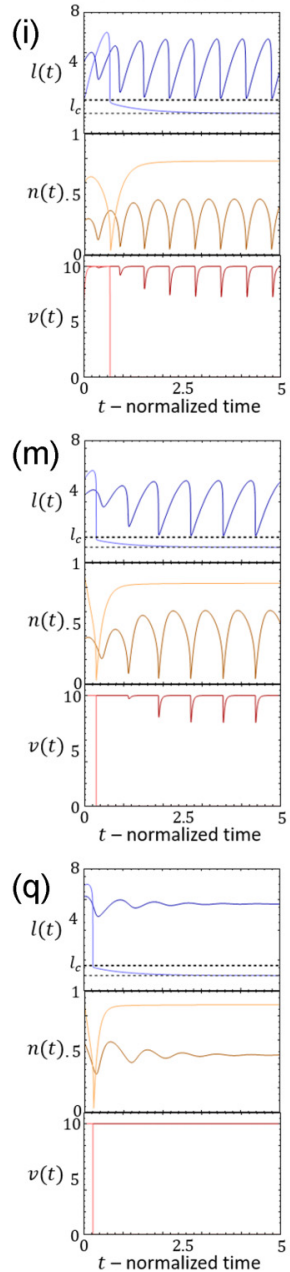

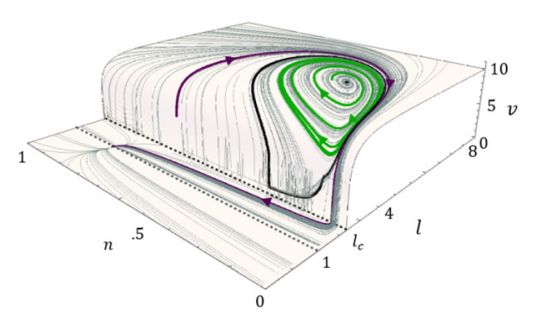

(n)

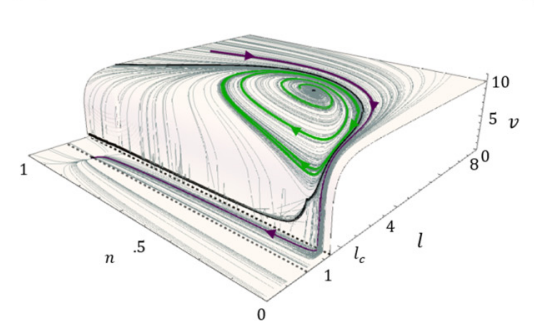

(r)

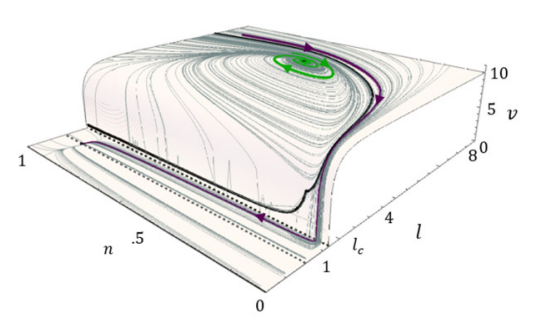

(g)
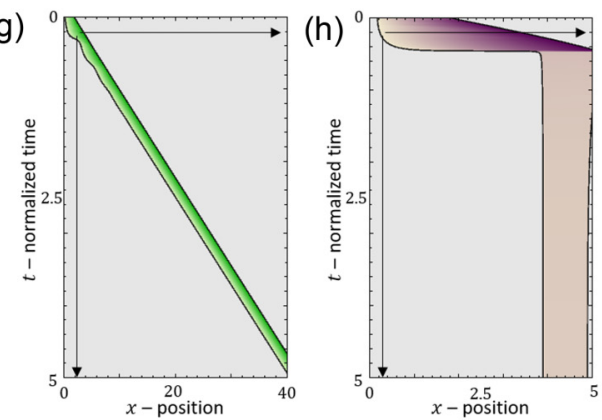

(k)
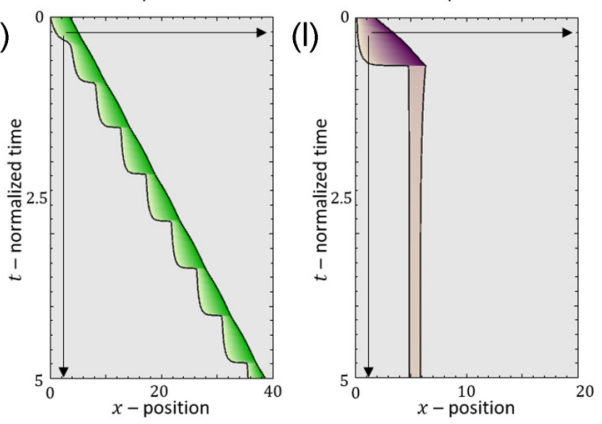

(o)
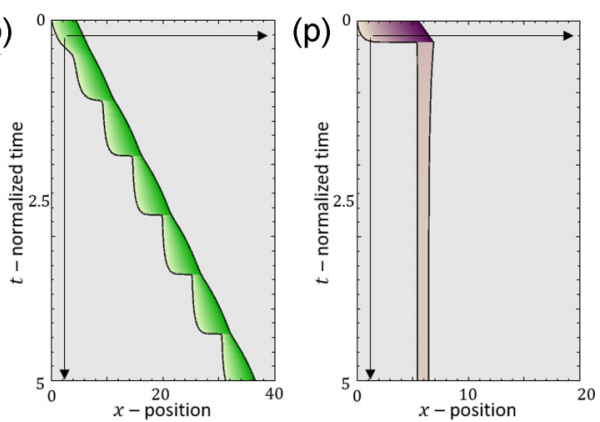

(s)
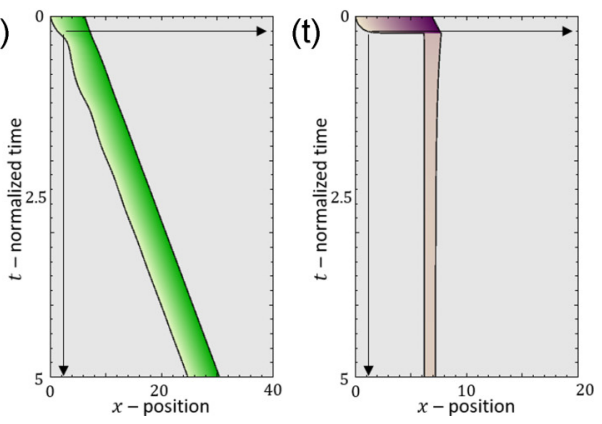

FIG. 26. The dynamics along the line of constant $k=1.1$ [vertical dashed line in Fig. 25(a)]. [(a)-(d)] $r=0.5$, [(e)-(h)] 1.5, [(i)-(l)] 3.5, $[(\mathrm{m})-(\mathrm{p})]$ 5, and [(q)-(t)] 8. Blue/orange/red curves in (a), (e), (i), (m), and (q) correspond to the time series of the cell length, adhesion concentration, and actin retrograde flow, respectively (bold/thin lines corresponds to green/purple trajectory). Green and purple curves in (b), (f), (j), (n), and (r) demonstrate the trajectories in the $l-n-v$ phase space. Black solid curves are the separatrices. (d), (g), and (i) display the corresponding kymographs. Parameters: $f_{s}=5, \kappa=20, \beta=11, c=3.85$, and $D=3.85$. 

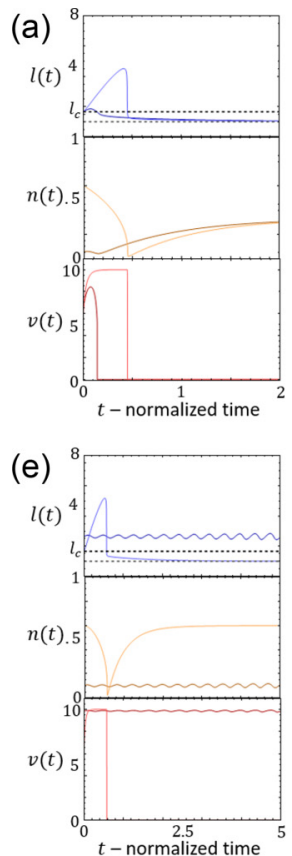

(i)

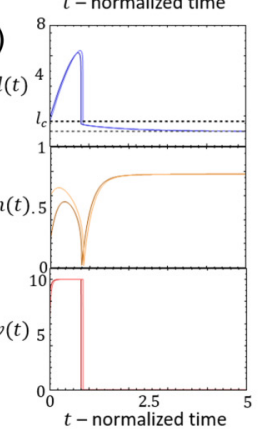

(m)
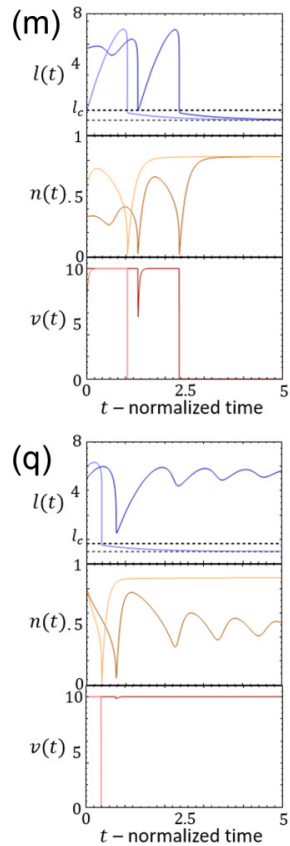

(b)

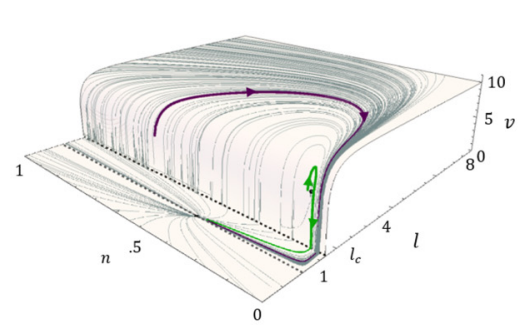

(f)

(c)
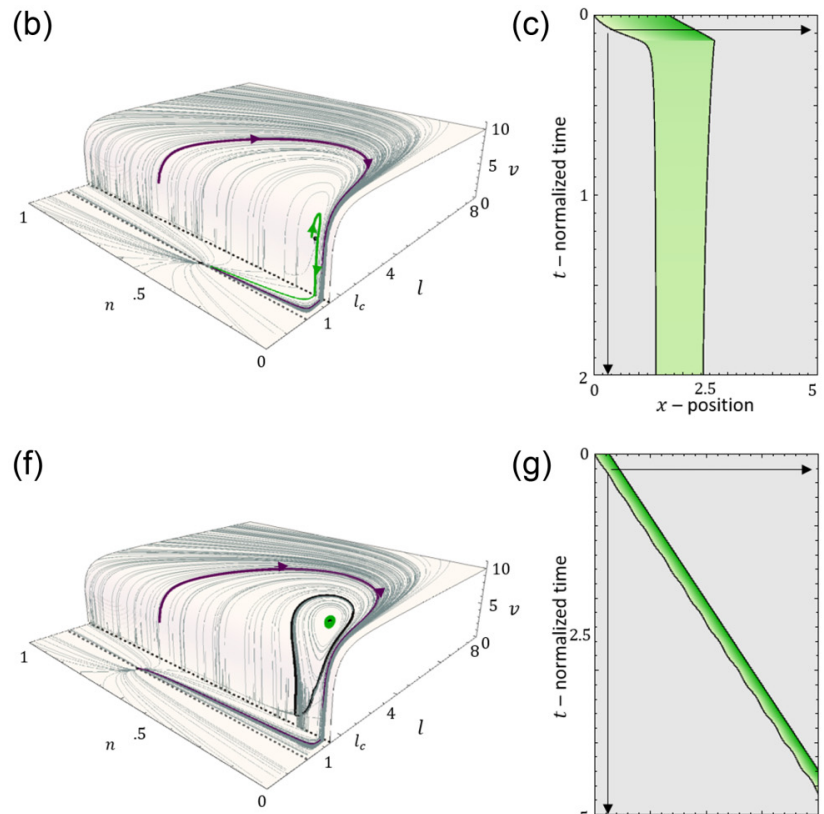

(j)

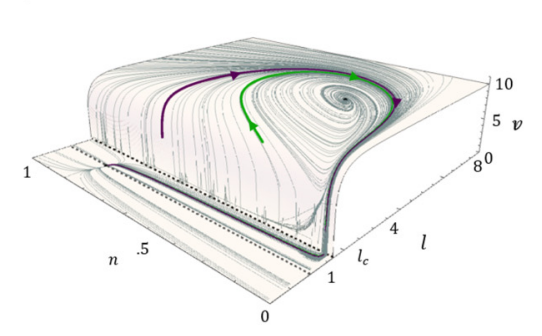

(n)

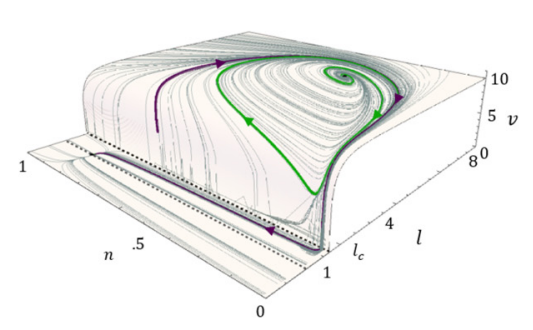

(r)

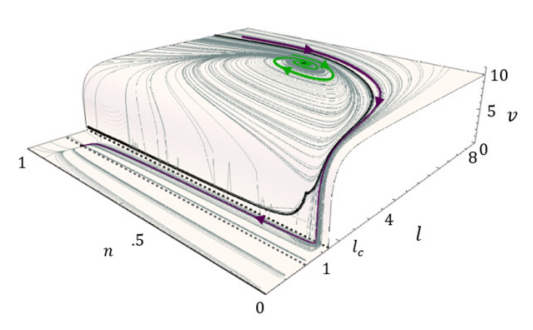

(g)

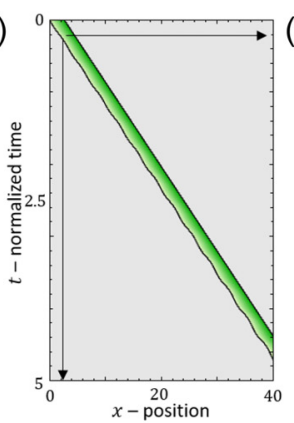

(k)

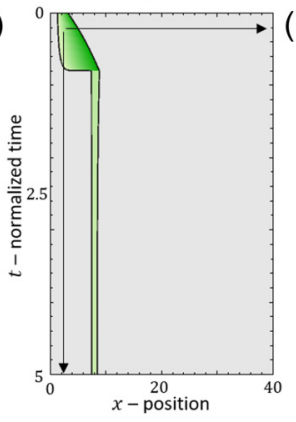

(o)

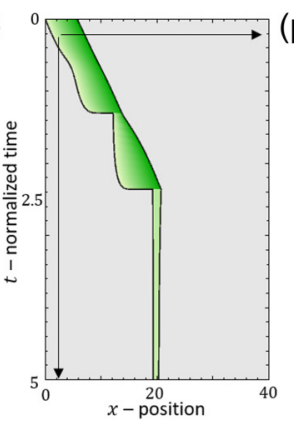

(s)

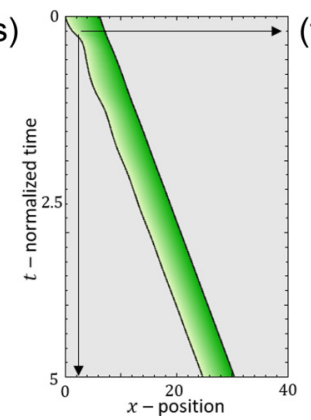

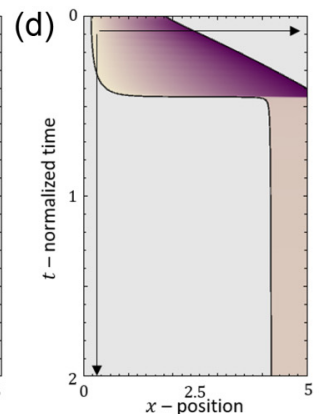
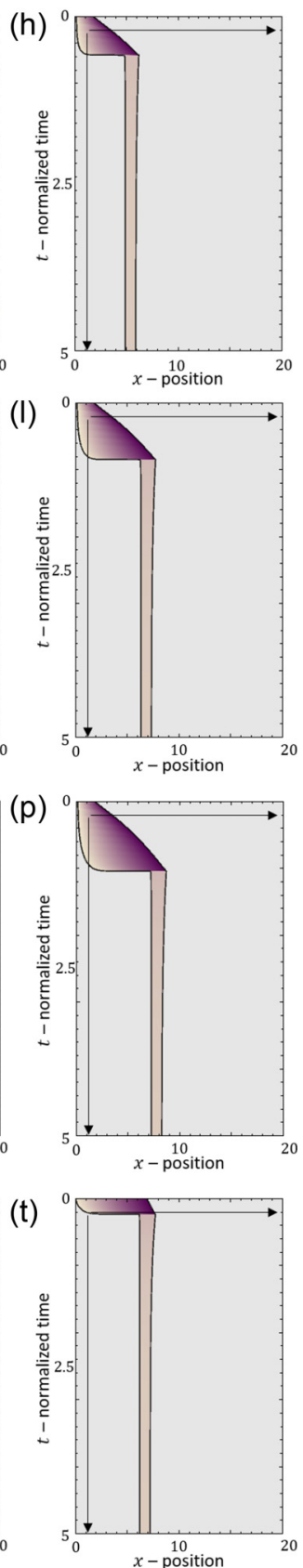

FIG. 27. The dynamics along the line of constant $k=0.8$ [vertical dashed line in Fig. 25(a)]. [(a)-(d)] $r=0.5$, [(e)-(h)] 1.5, [(i)-(1)] 3.5, $[(\mathrm{m})-(\mathrm{p})] 5$, and [(q)-(t)] 8. Blue/orange/red curves in (a), (e), (i), (m), and (q) correspond to the time series of the cell length, adhesion concentration and actin retrograde flow respectively (bold/thin lines corresponds to green/purple trajectory). Green and purple curves in (b), (f), (j), (n), and (r) demonstrate the trajectories in the $l-n-v$ phase space. Black solid curves are the separatrices. (d), (g), and (i) display the corresponding kymographs. Parameters: $f_{s}=5, \kappa=20, \beta=11, c=3.85$, and $D=3.85$. 


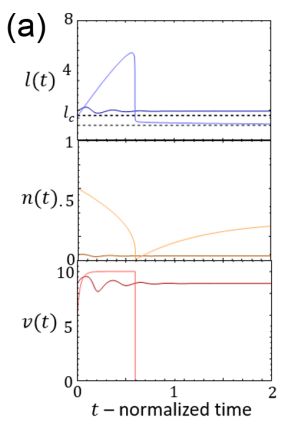

(b)

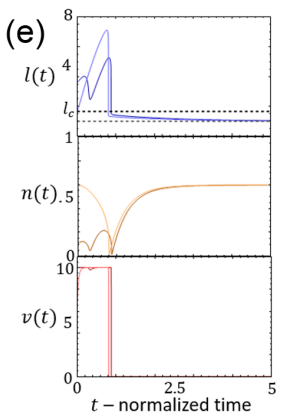

(i)
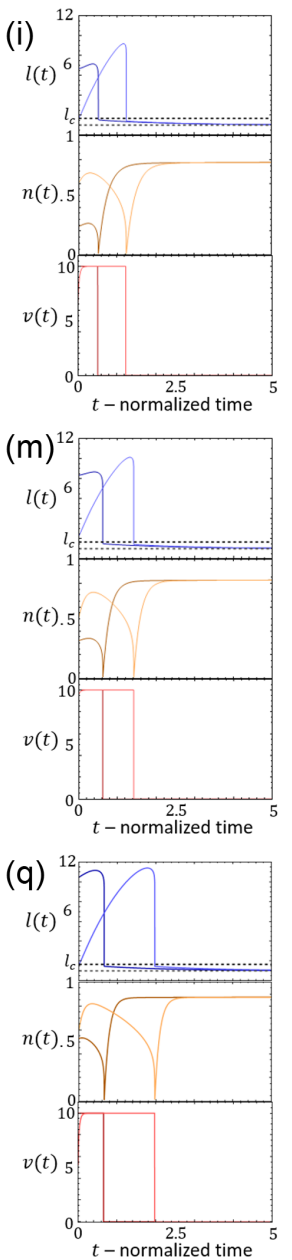

(f)

(j)

(n)

$(r)$
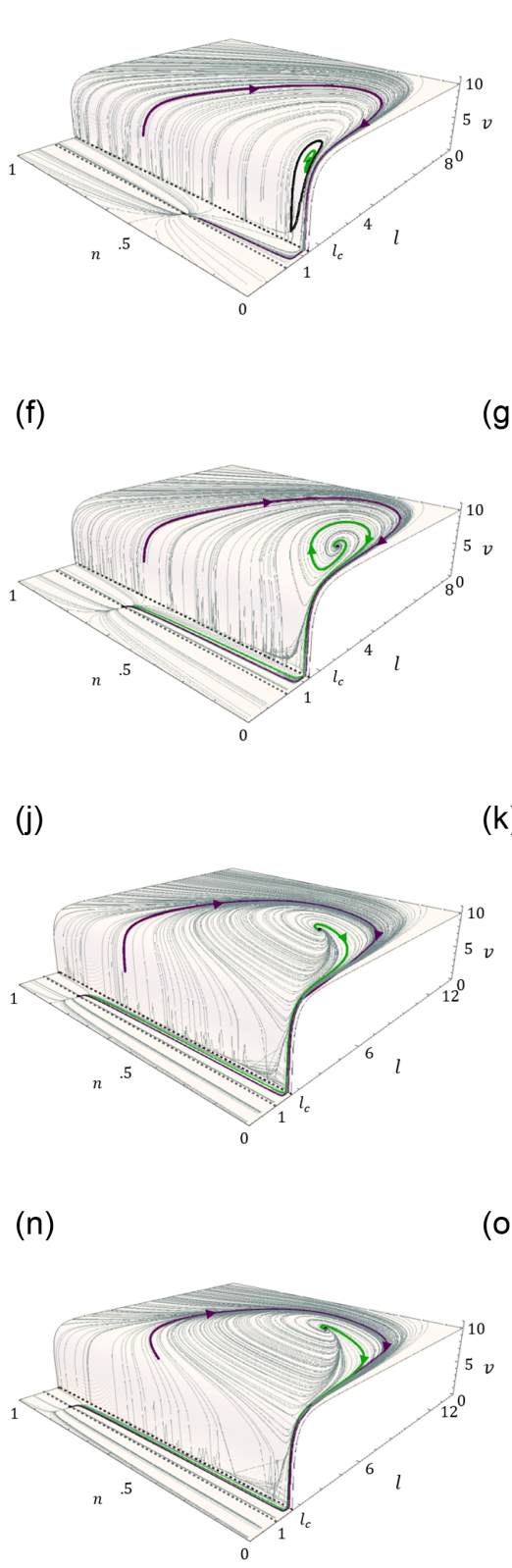

(o)
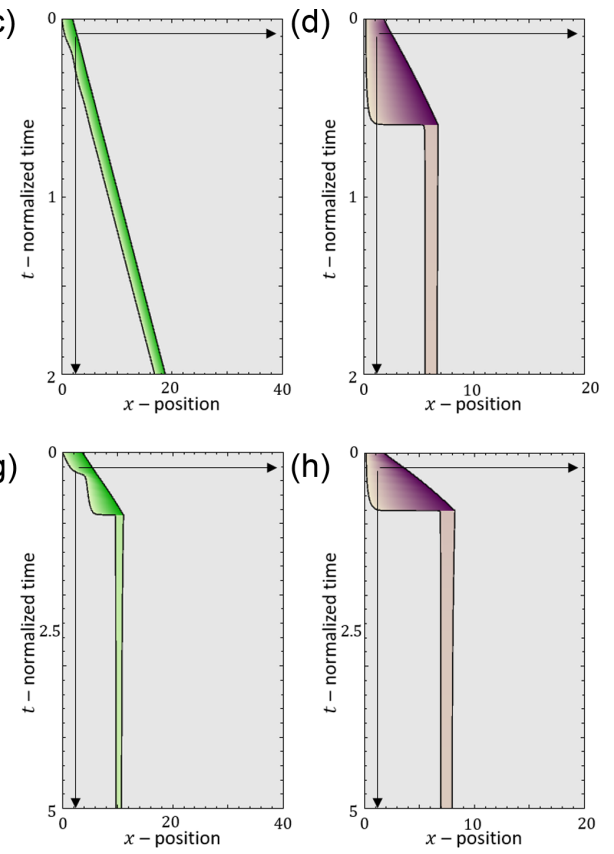

(k)
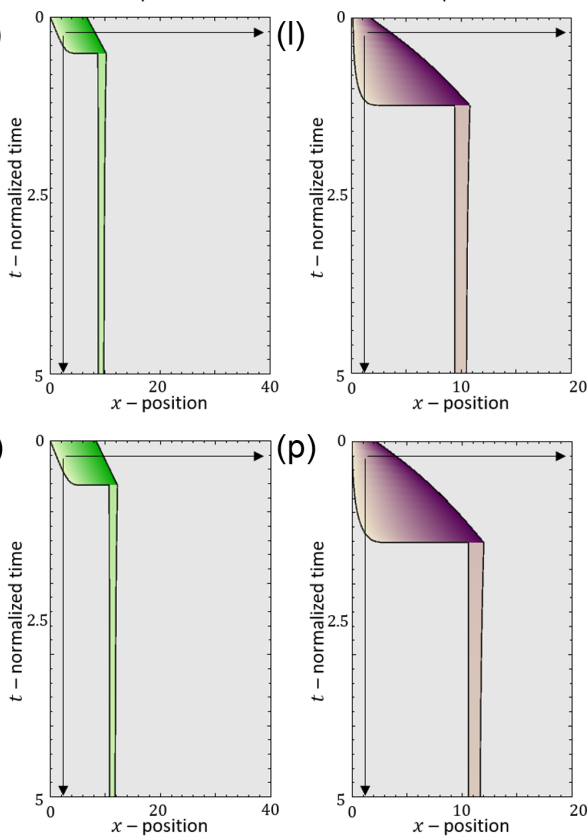

(s)
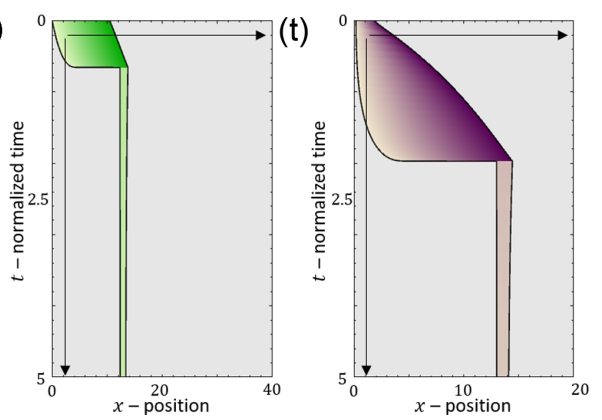

FIG. 28. The dynamics along the line of constant $k=0.5$ [vertical dashed line in Fig. 25(a)]. [(a)-(d)] $r=0.5$, [(e)-(h)] 1.5, [(i)-(l)] 3.5, $[(\mathrm{m})-(\mathrm{p})] 5$, and $[(\mathrm{q})-(\mathrm{t})]$ 8. Blue/orange/red curves in (a), (e), (i), (m), and (q) correspond to the time series of the cell length, adhesion concentration and actin retrograde flow respectively (bold/thin lines corresponds to green/purple trajectory). Green and purple curves in (b), (f), (j), (n), and (r) demonstrate the trajectories in the $l-n-v$ phase space. Black solid curves are the separatrices. (d), (g), and (i) display the corresponding kymographs. Parameters: $f_{s}=5, \kappa=20, \beta=11, c=3.85$, and $D=3.85$. 
cue acts on both ends of the cell, and inhibits actin polymerization.

The derivation starts by dividing the cue into two populations: (1) bound cue proteins $c_{b}$ which are advected by the retrograde flow $v$ and (2) unbound cue proteins $c_{f}$ which diffuse with a diffusion coefficient $D$.

The bound/unbound proteins can attach/detach to/from the advected actin, with rates $\bar{k}_{\text {on }} / \bar{k}_{\text {off }}$, respectively. The total concentration of the polarity cue in the cell is considered as conserved, i.e., $c_{\text {tot }}=c_{b}(x, t)+c_{f}(x, t) \quad \forall$ $\{t, x\}$.

The advection-diffusion transport equations of $c_{b}$ and $c_{f}$ are given by

$$
\begin{gathered}
\frac{\partial c_{b}(x, t)}{\partial t}=v \frac{\partial c_{b}(x, t)}{\partial x}+\bar{k}_{\mathrm{on}} c_{f}(x, t)-\bar{k}_{\mathrm{off}} c_{b}(x, t), \\
\frac{\partial c_{f}(x, t)}{\partial t}=D \frac{\partial^{2} c_{f}(x, t)}{\partial x^{2}}-\bar{k}_{\mathrm{on}} c_{f}(x, t)+\bar{k}_{\mathrm{off}} c_{b}(x, t) .
\end{gathered}
$$

At the limit of fast exchange, in which the kinetic rates $\bar{k}_{\text {on }}, \bar{k}_{\text {off }} \gg \frac{D}{\delta x^{2}}, \frac{v}{\delta x}$, along any segment $\delta x \in[0, L]$ [Eqs. (E1) and $(\mathrm{E} 2)]$ reduce to

$$
\frac{\partial c(x, t)}{\partial t}\left(1+\frac{\bar{k}_{\text {on }}}{\bar{k}_{\text {off }}}\right)=\frac{\partial}{\partial x}\left(v c(x, t)+\frac{\bar{k}_{\text {on }}}{\bar{k}_{\text {off }}} D \frac{\partial c(x, t)}{\partial x}\right),
$$

where $c(x, t) \equiv c_{b}(x, t)$.

At steady state, Eq. (E3) takes the form of

$$
0=\frac{\partial}{\partial x}\left(v c(x)+D \frac{\partial c(x, t)}{\partial x}\right)
$$

where $\frac{\bar{k}_{\text {on }}}{\bar{k}_{\text {off }}} D \rightarrow D$.

The solution of Eq. (E4) reads

$$
c(x)=c_{0} e^{-\frac{v x}{D}}+c_{1}
$$

and the coefficients are obtained by applying a no-flux boundary condition at $x_{f}$ and $x_{b}$

$$
\begin{aligned}
& 0=\left(v+\dot{x}_{b}\right) c(x)+\left.D \frac{\partial c(x)}{\partial x}\right|_{x=x_{b}}, \\
& 0=\left(v-\dot{x}_{f}\right) c(x)+\left.D \frac{\partial c(x)}{\partial x}\right|_{x=x_{f}} .
\end{aligned}
$$

We simplify the analysis by working in the regime where $\dot{x}_{b, f} \ll v$, so that in Eqs. (E6) and (E7), we neglect these terms and assume that the cell ends are quasi-stationary. Since we solve $c(x)$ in the cell frame, this quasistationary condition applies when the rate of cell-length change is slow: $i \ll v$. For cells moving at constant length, this is clearly valid, while during the stick-slip cycle (except for the fast slip event) we find that $l / v<\sim 0.3$.
By considering mass conservation, we obtain

$$
c_{\text {tot }}=c_{0} \int_{x_{b}}^{x_{f}} e^{-\frac{v x}{D}} d x \rightarrow c_{0}=\frac{c_{\text {tot }} v}{D}\left(\frac{1}{e^{-\frac{v x_{b}}{D}}-e^{-\frac{v x_{f}}{D}}}\right)
$$

and therefore the concentration profile is given by Eq. (19).

\section{APPENDIX F: RESCALING THE VELOCITY EQUATION IN THE ASYMMETRIC SELF-POLARIZED MODEL}

$$
\begin{aligned}
& v_{s s}\left(x_{f}, x_{b}\right) \\
& =\beta\left(\tilde{c}\left(x_{f}\right)-\tilde{c}\left(x_{b}\right)\right)=\beta\left(\frac{c_{s}}{c_{s}+c\left(x_{f}\right)}-\frac{c_{s}}{c_{s}+c\left(x_{b}\right)}\right) \\
& =\beta\left(\frac{1}{1+\frac{c_{\mathrm{tot}}}{c_{s}} \frac{v}{D}\left(\frac{e^{-\frac{v x_{f}}{D}}}{e^{-\frac{v x_{b}}{D}}-e^{-\frac{v x_{f}}{D}}}\right)}-\frac{1}{1+\frac{c_{\mathrm{tot}}}{c_{s}} \frac{v}{D}\left(\frac{e^{-\frac{v x_{b}}{D}}}{e^{-\frac{v x_{b}}{D}}-e^{-\frac{v x_{f}}{D}}}\right)}\right) .
\end{aligned}
$$

Next, we rescale Eq. (20) by the time and length scale $k_{\text {off }}^{-1}$ and $x_{0}$, such that $\frac{x_{0}^{2}}{k_{\text {off }}^{-1}} D \rightarrow D, \frac{k_{\text {off }}^{-1} v}{x_{0}} \rightarrow v$, and $\frac{k_{\text {off }}^{-1} \beta}{x_{0}} \rightarrow \beta \frac{c_{\text {tot }} x_{0}}{c_{\mathrm{s}}} \rightarrow c$, and then rescale $x_{0} x_{f} \rightarrow x_{f}, x_{0} x_{b} \rightarrow x_{b}$, and $\frac{c_{\text {tot }}}{c_{\mathrm{s}} x_{0}} \rightarrow c$ which gives

$$
\begin{aligned}
& v_{s s}\left(x_{f}, x_{b}\right) \\
& =\beta\left(\frac{1}{1+c \frac{v}{D}\left(\frac{e^{-\frac{v x_{f}}{D}}}{e^{-\frac{v x_{b}}{D}}-e^{-\frac{v x_{f}}{D}}}\right)}-\frac{1}{1+c \frac{v}{D}\left(\frac{e^{-\frac{v x_{b}}{D}}}{e^{-\frac{v x_{b}}{D}}-e^{-\frac{v x_{f}}{D}}}\right)}\right) \\
& =\beta\left(\frac{1}{1+c \frac{v}{D}\left(\frac{1}{e^{\frac{v\left(x_{f}-x_{b}\right)}{D}}-1}\right)}-\frac{1}{1+c \frac{v}{D}\left(\frac{1}{1-e^{-\frac{v\left(x_{f}-x_{b}\right)}{D}}}\right)}\right)
\end{aligned}
$$

and finally we change coordinates to $l=x_{f}-x_{b}$, and obtain Eq. (21).

\section{APPENDIX G: BIFURCATION ANALYSIS}

The introduction of the actin dynamics in Sec. IIB, introduces to the $n-l$ vector field a discontinuity at $l=l_{c}$. The vector discontinuity gives rise to an additional unstable limit cycle due to a trajectory that collides with the line of $l=l_{c}$ (Figs. 8 and 9). The period of the limit cycle is finite as long as the amplitude of the $n$ coordinate is limited to values: $n<\frac{r}{1+r}$. When the $n$ coordinate amplitude grows beyond this limit, $n>\frac{r}{1+r}$, the period of the discontinuous unstable limit cycle is infinite (Figs. 25-28).
[1] D. I. Shreiber, V. H. Barocas, and R. T. Tranquillo, Temporal variations in cell migration and traction during fibroblastmediated gel compaction, Biophys. J. 84, 4102 (2003).

[2] K. Keren, Z. Pincus, G. M. Allen, E. L. Barnhart, G. Marriott, A. Mogilner, and J. A. Theriot, Mechanism of shape determination in motile cells, Nature (London) 453, 475 (2008).
[3] H. Takagi, M. J. Sato, T. Yanagida, and M. Ueda, Functional analysis of spontaneous cell movement under different physiological conditions, PLoS One 3, e2648 (2008).

[4] P.-H. Wu, A. Giri, S. X. Sun, and D. Wirtz, Three-dimensional cell migration does not follow a random walk, Proc. Natl. Acad. Sci. USA 111, 3949 (2014). 
[5] J. M. Kowalewski, H. Shafqat-Abbasi, M. Jafari-Mamaghani, B. E. Ganebo, X. Gong, S. Strömblad, and J. G. Lock, Disentangling membrane dynamics and cell migration; differential influences of f-actin and cell-matrix adhesions, PLoS One 10, e0135204 (2015).

[6] D. B. Brückner, A. Fink, J. O. Rädler, and C. P. Broedersz, Disentangling the behavioural variability of confined cell migration, J. R. Soc., Interface 17, 20190689 (2020).

[7] R. J. Petrie, A. D. Doyle, and K. M. Yamada, Random versus directionally persistent cell migration, Nat. Rev. Mol. Cell Biol. 10, 538 (2009).

[8] Y. Miao, S. Bhattacharya, M. Edwards, H. Cai, T. Inoue, P. A. Iglesias, and P. N. Devreotes, Altering the threshold of an excitable signal transduction network changes cell migratory modes, Nature Cell Biology 19, 329 (2017).

[9] P. Monzo, Y. K. Chong, C. Guetta-Terrier, A. Krishnasamy, S. R. Sathe, E. K. Yim, W. H. Ng, B. T. Ang, C. Tang, B. Ladoux et al., Mechanical confinement triggers glioma linear migration dependent on formin fhod3, Mol. Biol. Cell 27, 1246 (2016).

[10] K. Hennig, I. Wang, P. Moreau, L. Valon, S. DeBeco, M. Coppey, Y. Miroshnikova, C. Albiges-Rizo, C. Favard, R. Voituriez et al., Stick-slip dynamics of cell adhesion triggers spontaneous symmetry breaking and directional migration of mesenchymal cells on one-dimensional lines, Sci. Adv. 6, eaau5670 (2020).

[11] L. Han, Z. Mao, J. Wu, Y. Guo, T. Ren, and C. Gao, Unidirectional migration of single smooth muscle cells under the synergetic effects of gradient swelling cue and parallel groove patterns, Colloids Surf., B 111, 1 (2013).

[12] K. M. Stroka, H. Jiang, S.-H. Chen, Z. Tong, D. Wirtz, S. X. Sun, and K. Konstantopoulos, Water permeation drives tumor cell migration in confined microenvironments, Cell 157, 611 (2014).

[13] M. Chabaud, M. L. Heuzé, M. Bretou, P. Vargas, P. Maiuri, P. Solanes, M. Maurin, E. Terriac, M. Le Berre, D. Lankar et al., Cell migration and antigen capture are antagonistic processes coupled by myosin ii in dendritic cells, Nat. Commun. 6, 7526 (2015).

[14] K. Sheets, S. Wunsch, C. Ng, and A. S. Nain, Shape-dependent cell migration and focal adhesion organization on suspended and aligned nanofiber scaffolds, Acta Biomater. 9, 7169 (2013).

[15] C. Guetta-Terrier, P. Monzo, J. Zhu, H. Long, L. Venkatraman, Y. Zhou, P. Wang, S. Y. Chew, A. Mogilner, B. Ladoux et al., Protrusive waves guide $3 \mathrm{~d}$ cell migration along nanofibers, J. Cell Biol. 211, 683 (2015).

[16] P. Friedl and D. Gilmour, Collective cell migration in morphogenesis, regeneration and cancer, Nat. Rev. Mol. Cell Biol. 10, 445 (2009).

[17] P. Rakic, Principles of neural cell migration, Experientia 46, 882 (1990).

[18] J. G. Parnavelas, The origin and migration of cortical neurones: New vistas, Trends Neurosci. 23, 126 (2000).

[19] C. D. Paul, P. Mistriotis, and K. Konstantopoulos, Cancer cell motility: lessons from migration in confined spaces, Nat. Rev. Cancer 17, 131 (2017).

[20] G. Danuser, J. Allard, and A. Mogilner, Mathematical modeling of eukaryotic cell migration: Insights beyond experiments, Annu. Rev. Cell Dev. Biol. 29, 501 (2013).
[21] D. Selmeczi, L. Li, L. I. I. Pedersen, S. F. Nrrelykke, P. H. Hagedorn, S. Mosler, N. B. Larsen, E. C. Cox, and H. Flyvbjerg, Cell motility as random motion: A review, Eur. Phys. J. Spec. Top. 157, 1 (2008).

[22] C. L. Vestergaard, J. N. Pedersen, K. I. Mortensen, and H. Flyvbjerg, Estimation of motility parameters from trajectory data, Eur. Phys. J.: Spec. Top. 224, 1151 (2015).

[23] C. E. Chan and D. J. Odde, Traction dynamics of filopodia on compliant substrates, Science 322, 1687 (2008).

[24] P. Sens, Rigidity sensing by stochastic sliding friction, Europhys. Lett. 104, 38003 (2013).

[25] P. Sens, Stick-slip model for actin-driven cell protrusions, cell polarization and crawling, arXiv:2006.00122.

[26] P. Maiuri, J.-F. Rupprecht, S. Wieser, V. Ruprecht, O. Bénichou, N. Carpi, M. Coppey, S. De Beco, N. Gov, C.-P. Heisenberg et al., Actin flows mediate a universal coupling between cell speed and cell persistence, Cell 161, 374 (2015).

[27] I. Lavi, M. Piel, A.-M. Lennon-Duménil, R. Voituriez, and N. S. Gov, Deterministic patterns in cell motility, Nat. Phys. 12, 1146 (2016).

[28] A. Giese, B. Laube, S. Zapf, U. Mangold, and M. Westphal, Glioma cell adhesion and migration on human brain sections, Anticancer Res. 18, 2435 (1998).

[29] K. J. Wolf, J. Chen, J. D. Coombes, M. K. Aghi, and S. Kumar, Dissecting and rebuilding the glioblastoma microenvironment with engineered materials, Nat. Rev. Mater. 4, 651 (2019).

[30] T. Y.-C. Tsai, S. R. Collins, C. K. Chan, A. Hadjitheodorou, P.-Y. Lam, S. S. Lou, H. W. Yang, J. Jorgensen, F. Ellett, D. Irimia et al., Efficient front-rear coupling in neutrophil chemotaxis by dynamic myosin ii localization, Dev. Cell 49, 189 (2019).

[31] J. A. Broussard, D. J. Webb, and I. Kaverina, Asymmetric focal adhesion disassembly in motile cells, Curr. Opin. Cell Biol. 20, 85 (2008).

[32] R. Rezk, B. Z. Jia, A. Wendler, I. Dimov, C. Watts, A. E. Markaki, K. Franze, and A. J. Kabla, Spatial heterogeneity of cell-matrix adhesive forces predicts human glioblastoma migration, bioRxiv: 10.1101/2020.05.06.080804.

[33] E. L. Barnhart, J. Allard, S. S. Lou, J. A. Theriot, and A. Mogilner, Adhesion-dependent wave generation in crawling cells, Curr. Biol. 27, 27 (2017).

[34] I. Dang, R. Gorelik, C. Sousa-Blin, E. Derivery, C. Guérin, J. Linkner, M. Nemethova, J. G. Dumortier, F. A. Giger, T. A. Chipysheva et al., Inhibitory signaling to the arp2/3 complex steers cell migration, Nature (London) 503, 281 (2013).

[35] T. Putelat, P. Recho, and L. Truskinovsky, Mechanical stress as a regulator of cell motility, Phys. Rev. E 97, 012410 (2018).

[36] A. D. Doyle, F. W. Wang, K. Matsumoto, and K. M. Yamada, One-dimensional topography underlies three-dimensional fibrillar cell migration, J. Cell Biol. 184, 481 (2009).

[37] P. Friedl, E. Sahai, S. Weiss, and K. M. Yamada, New dimensions in cell migration, Nat. Rev. Mol. Cell Biol. 13, 743 (2012).

[38] I. Lavi, N. Meunier, R. Voituriez, and J. Casademunt, Motility and morphodynamics of confined cells, Phys. Rev. E 101, 022404 (2020).

[39] E. Barnhart, K.-C. Lee, G. M. Allen, J. A. Theriot, and A. Mogilner, Balance between cell- substrate adhesion and myosin contraction determines the frequency of motility initiation in 
fish keratocytes, Proc. Natl. Acad. Sci. U.S.A. 112, 5045 (2015).

[40] S. Jain, V. M. Cachoux, G. H. N. S. Narayana, S. de Beco, J. D’Alessandro, V. Cellerin, T. Chen, M. L. Heuzé, P. Marcq, R.-M. Mège et al., The role of single-cell mechanical behaviour and polarity in driving collective cell migration, Nat. Phys. 16, $802(2020)$

[41] P. Monzo, M. Crestani, Y. K. Chong, K. Hennig, A. Ghisleni, Q. Li, C. Richichi, P. Maiuri, M. Balland, M. P. Sheetz, G. Pelicci, B. T. Ang, C. Tang, and N. C. Gauthier, Adaptive mechanoproperties characterize glioblastoma fitness for invasion, bioRxiv:10.1101/2020.06.17.156406.
[42] B. L. Bangasser, S. S. Rosenfeld, and D. J. Odde, Determinants of maximal force transmission in a motor-clutch model of cell traction in a compliant microenvironment, Biophys. J. 105, 581 (2013).

[43] Y.-K. Chong, T.-B. Toh, N. Zaiden, A. Poonepalli, S. H. Leong, C. E. L. Ong, Y. Yu, P. B. Tan, S.-J. See, W.-H. Ng et al., Cryopreservation of neurospheres derived from human glioblastoma multiforme, Stem Cells 27, 29 (2009).

[44] A. Azioune, N. Carpi, Q. Tseng, M. Thery, and M. Piel, Protein micropatterns: A direct printing protocol using deep UVs, in Methods in Cell Biology (Academic Press, 2010), Vol. 97, pp. 133-146. 